\title{
OPTIMAL BILINEAR CONTROL OF NONLINEAR STOCHASTIC SCHRÖDINGER EQUATIONS DRIVEN BY LINEAR MULTIPLICATIVE NOISE ${ }^{1}$
}

\author{
BY VIOREL BARBU* ${ }^{*} \dagger, 2$, MiChAEL RÖCKNER ${ }^{\ddagger}$ AND DENG ZHANG ${ }^{\ddagger}, \S, 3,4$ \\ Octav Mayer Institute of Mathematics (Romanian Academy)*, Al.I. Cuza \\ University $^{\dagger}$, Universität Bielefeld ${ }^{\ddagger}$ and Shanghai Jiao Tong University ${ }^{\S}$
}

We analyze the bilinear optimal control problem of quantum mechanical systems with final observation governed by a stochastic nonlinear Schrödinger equation perturbed by a linear multiplicative Wiener process. The existence of an open-loop optimal control and first-order Lagrange optimality conditions are derived, via Skorohod's representation theorem, Ekeland's variational principle and the existence for the linearized dual backward stochastic equation. Moreover, our approach in particular applies to the deterministic case.

1. Introduction. We consider the controlled stochastic system governed by the nonlinear Schrödinger equation:

$$
\begin{aligned}
i d X(t, \xi)= & \Delta X(t, \xi) d t+\lambda|X(t, \xi)|^{\alpha-1} X(t, \xi) d t-i \mu(\xi) X(t, \xi) d t \\
& +V_{0}(\xi) X(t, \xi) d t+\sum_{j=1}^{m} u_{j}(t) V_{j}(\xi) X(t, \xi) d t \\
& +i X(t, \xi) d W(t, \xi), \quad t \in(0, T), \xi \in \mathbb{R}^{d}, \\
X(0)= & x \quad \text { in } \mathbb{R}^{d} .
\end{aligned}
$$

Here, $\lambda= \pm 1, \alpha>1, V_{j} \in W^{1, \infty}\left(\mathbb{R}^{d}\right), 0 \leq j \leq m$, are real valued functions, $W$ is the Wiener process,

$$
W(t, \xi)=\sum_{j=1}^{N} \mu_{j} e_{j}(\xi) \beta_{j}(t), \quad t \geq 0, \xi \in \mathbb{R}^{d}
$$

Received July 2016; revised July 2017.

${ }^{1}$ Supported by the DFG through SFB 1283.

${ }^{2}$ Supported by a grant of Ministry of Research and Innovation, CNCS-UEFISCDI, project number PN-III-P4-ID-PCE-2016-0011, within PNCDI III.

${ }^{3}$ Supported by NSFC (No. 11501362).

${ }^{4}$ Corresponding author.

MSC2010 subject classifications. 60H15, 35Q40, 49K20, 35J10.

Key words and phrases. Backward stochastic equation, nonlinear Schrödinger equation, optimal control, Wiener process. 
and

$$
\begin{aligned}
& u(t)=\left(u_{1}(t), \ldots, u_{m}(t)\right) \in \mathbb{R}^{m}, \quad t \in(0, T), \\
& \mu(\xi)=\frac{1}{2} \sum_{j=1}^{N}\left|\mu_{j}\right|^{2} e_{j}^{2}(\xi), \quad \xi \in \mathbb{R}^{d}, d \geq 1,
\end{aligned}
$$

with $\mu_{j}$ purely imaginary numbers (i.e., $\left.\operatorname{Re} \mu_{j}=0\right), e_{j}(\xi)$ real-valued functions and $\beta_{j}$ independent real Brownian motions on a probability space $(\Omega, \mathcal{F}, \mathbb{P})$ with natural filtration $\left(\mathcal{F}_{t}\right)_{t \geq 0}, 1 \leq j \leq N$. For simplicity, we assume $N<\infty$, but the arguments in this paper easily extend to the case where $N=\infty$.

The physical significance of (1.1) is well known. $X=X(t, \xi, \omega), \xi \in \mathbb{R}^{d}, t \geq 0$, $\omega \in \Omega$, represents the quantum state at time $t$, while the stochastic perturbation $i X d W$ represents a stochastic continuous measurement via the pointwise quantum observables $R_{j}(X)=\mu_{j} e_{j} X$. The function $V_{0}$ describes an external potential.

In the conservative case considered in this paper (i.e., $\operatorname{Re} \mu_{j}=0,1 \leq j \leq N$ ), $-i \mu X d t+i X d W$ is indeed the Stratonovitch differential. It follows by Itô's formula that $|X(t)|_{2}^{2}=|x|_{2}^{2}, \forall t \geq 0$. Hence, normalizing the initial state $|x|_{2}=1$, we have $|X(t)|_{2}=1, \forall t \in[0, T]$, and so, the quantum system evolves on the unit ball of $L^{2}$ and verifies the conservation of probability; see, for example, [9, 10].

We also mention that, for the general case when $\mu_{j}$ are complex numbers, one of the main features is that the mean norm square $|X(t)|_{2}^{2}, t \in[0, T]$, is a continuous martingale. This fact enables one to define the "physical" probability law and implies the conservation of $\mathbb{E}|X(t)|_{2}^{2}, t \in[0, T]$, which plays an important role in the application to open quantum systems; see, for example, [4] for more details. See also $[1,2]$ for global well-posedness with exponents of the nonlinearity in the optimal subcritical range.

As regards the real valued input control $u$, in most situations it represents an external applied force due to the interaction of the quantum system with an electric field or a laser pulse applied to a quantum system.

Here, motivated by the optimal control of quantum mechanical systems (cf., e.g., [24, 29]), we shall study an optimal control problem associated with the control system (1.1) which, in a few words, can be described as follows [see Problem (P) below]: find an input control $u$ that steers in time $T$ the state $X$ as close as possible of a target state $\mathbb{X}_{T}$ and a given trajectory $\mathbb{X}_{1}$, and with a reasonable minimum energy. Roughly speaking, this means to find the quantum mechanical potential $u$ from observation of the quantum state $X(T)$ at the end of time interval $[0, T]$.

It should be mentioned that there is an extensive literature on the deterministic bilinear control equation (1.1) mainly concerned with exact controllability in time $T$ of Schrödinger equations or with the optimal control problem (see, for instance, $[5-7,16,18,19,23,24,29])$. However, there are very few results on optimal control problems governed by nonlinear Schrödinger equations (i.e., $\alpha>1$ ) and, to the best of our knowledge, none for stochastic control systems (1.1) with linear multiplicative noise. In the latter case, the existence of an optimal control is largely an 
open problem, since the cost functional is not simultaneously lower semicontinuous and coercive in the basic control space. It is also very interesting to consider the control in the diffusion coefficient (see, e.g., [30]). In this paper, motivated by the quantum control problem mentioned above, we will focus on the case where the control acts in the potential of the quantum mechanical system.

The approach we used here is based on Skorohod's representation theorem and Ekeland's variational principle, and this is one of the main novelties of this work. The approach is also based on an existence result of the linearized backward dual stochastic equation, which is also new in the literature and uses sharp stochastic estimates for linear Schrödinger equations with time dependent coefficients (see $[1,2])$. As a matter of fact, a great effort of this work is dedicated to this issue.

2. Formulation of problem and the main results. To begin with, we recall the definition of a strong solution to equation (1.1) (see [1, 2]).

Definition 2.1. Let $x \in L^{2}$ (resp., $H^{1}$ ), $0<T<\infty$. Let $\alpha$ satisfy $1<\alpha<$ $1+\frac{4}{d}$ [resp., $1<\alpha<1+\frac{4}{(d-2)_{+}}$] $d \geq 1$. A strong $L^{2}$-(resp., $H^{1}$-)solution to (1.1) on $[0, T]$ is an $L^{2}$-(resp., $H^{1}$-)valued continuous $\left(\mathcal{F}_{t}\right)_{t \geq 0}$-adapted process $X=X(t)$ such that $|X|^{\alpha-1} X \in L^{1}\left(0, T ; H^{-1}\right)$, and $\mathbb{P}$-a.s.,

$$
\begin{aligned}
X(t)= & x-\int_{0}^{t}\left(i \Delta X(s)+\mu X(s)+\lambda i|X(s)|^{\alpha-1} X(s, \xi)+i V_{0}(\xi) X(s)\right. \\
& \left.+i \sum_{j=1}^{m} u_{j}(s) V_{j}(\xi) X(s)\right) d s+\int_{0}^{t} X(s) d W(s), \quad t \in[0, T],
\end{aligned}
$$

as an Itô equation in $H^{-2}$ (resp., $\left.H^{-1}\right)$. Here, the controller $u=\left(u_{1}, \ldots, u_{m}\right)$ is in the admissible set $\mathcal{U}_{\mathrm{ad}}$, defined by

$$
\mathcal{U}_{\mathrm{ad}}=\left\{u \in L_{\mathrm{ad}}^{2}\left(0, T ; \mathbb{R}^{m}\right) ; u \in U, \text { a.e. on }(0, T) \times \Omega\right\},
$$

where $L_{\text {ad }}^{2}\left(0, T ; \mathbb{R}^{m}\right)$ is the space of all $\left(\mathcal{F}_{t}\right)_{t \geq 0}$-adapted $\mathbb{R}^{m}$-valued processes $u$ : $[0, T] \rightarrow \mathbb{R}^{m}$ such that $u \in L^{2}\left((0, T) \times \Omega ; \mathbb{R}^{m}\right)$, and $U$ is a compact convex subset of $\mathbb{R}^{m}$.

It is easy to check that $\int_{0}^{t} X(s) d W(s)$ in Definition 2.1 is an $L^{2}$-(resp., $H^{1}$-)valued continuous stochastic integral. (We refer, e.g., to [8, 21] for the general theory of infinite dimensional stochastic integrals.) Moreover, $\sup _{u \in \mathcal{U}_{\mathrm{ad}}}\|u\|_{L^{\infty}\left(0, T ; \mathbb{R}^{m}\right)} \leq D_{U}<\infty$, where $D_{U}$ is the diameter of $U$.

Following $[1,2]$, we introduce the hypotheses below.

(H0) $1<\alpha<1+\frac{4}{d}$. For each $1 \leq j \leq N, e_{j} \in C_{b}^{\infty}\left(\mathbb{R}^{d}\right)$ satisfies

$$
\lim _{|\xi| \rightarrow \infty} \zeta(\xi)\left|\partial^{\gamma} e_{j}(\xi)\right|=0
$$


where $\gamma$ is a multi-index such that $1 \leq|\gamma| \leq 2$, and

$$
\zeta(\xi)= \begin{cases}1+|\xi|^{2} & \text { if } d \neq 2, \\ \left(1+|\xi|^{2}\right)\left(\ln \left(3+|\xi|^{2}\right)\right)^{2} & \text { if } d=2 .\end{cases}
$$

(H1) In the defocusing case $\lambda=-1,1<\alpha<1+\frac{4}{(d-2)_{+}}$, and in the focusing case $\lambda=1,1<\alpha<1+\frac{4}{d}$. For each $1 \leq j \leq N, e_{j} \in C_{b}^{\infty}\left(\mathbb{R}^{d}\right)$ satisfies (2.3) for any multi-index $1 \leq|\gamma| \leq 3$.

The global existence, uniqueness and uniform estimates of the solution to (1.1) used in this paper are summarized in Proposition 2.2 below.

Proposition 2.2. Assume (H0) [resp., (H1)]. For each $x \in L^{2}$ (resp., $H^{1}$ ), $u \in \mathcal{U}_{\mathrm{ad}}$ and $0<T<\infty$, there exists a unique strong $L^{2}$-(resp., $H^{1}$-)solution $X^{u}$ to (1.1), satisfying $|X(t)|_{2}=|x|_{2}, t \in[0, T]$ (resp., for any $\rho \geq 1$,

$$
\left.\sup _{u \in \mathcal{U}_{\mathrm{ad}}} \mathbb{E}\left\|X^{u}\right\|_{C\left([0, T] ; H^{1}\right)}^{\rho}<\infty\right) .
$$

Moreover, assuming that the exponent $\alpha$ is in the range specified in (H1) and that $e_{k}$ are constants, $1 \leq k \leq N$, we have for any $\rho \geq 1$,

$$
\sup _{u \in \mathcal{U}_{\mathrm{ad}}}\left(\left\|X^{u}\right\|_{L^{\infty}\left(\Omega ; L^{q}\left(0, T ; L^{p}\right)\right)}+\left\|X^{u}\right\|_{L^{\rho}\left(\Omega ; L^{q}\left(0, T ; W^{1, p}\right)\right)}\right)<\infty,
$$

where $(p, q)$ is any Strichartz pair, that is, $\frac{2}{q}=\frac{d}{2}-\frac{d}{p},(p, q) \in[2, \infty] \times[2, \infty]$, and $(p, q, d) \neq(\infty, 2,2)$.

The global existence and uniqueness can be proved similarly as in $[1,2]$ by the rescaling approach and the Strichartz estimates for lower order perturbations of the Laplacian. We refer to [1], Lemma 4.1 and [2], Lemma 2.7, for explicit formulations of Strichartz estimates in the $L^{p}$ and Sobolev spaces, respectively. The technical proof of the estimates (2.4) and (2.5) is postponed to the Appendix for simplicity of the exposition.

We mention that, for controlled stochastic Schrödinger equations with control also in the diffusion coefficient, that is, $i X d W$ and $\mu$ in (1.1) are replaced by $i X \sum_{j=1}^{N} \sigma_{j}(u) \mu_{j} e_{j} d \beta_{j}(t)$ and $1 / 2 \sum_{j=1}^{N}\left(\sigma_{j}(u)\right)^{2}\left|\mu_{j}\right|^{2} e_{j}^{2}$, respectively, one also has global well-posedness by using the rescaling transformation $y=e^{-\Phi(W)} X$, where $\Phi(W)=\sum_{j=1}^{N} \int_{0}^{t} \sigma(u(s)) \mu_{j} e_{j} d \beta_{j}(s)$. However, the optimal control problem is technically more difficult; see Remark 2.9 below.

In the following, $L_{\text {ad }}^{2}\left(0, T ; L^{2}\left(\Omega ; L^{2}\right)\right)$ is the space of $L^{2}$-valued $\left(\mathcal{F}_{t}\right)_{t \geq 0^{-}}$ adapted processes $u$ such that $\mathbb{E} \int_{0}^{T}|u(t)|_{2}^{2} d t<\infty$.

The optimal control problem we study in the following is 
(P) Minimize

$\mathbb{E}\left(\left|X(T)-\mathbb{X}_{T}\right|_{2}^{2}+\gamma_{1} \int_{0}^{T}\left|X(t)-\mathbb{X}_{1}(t)\right|_{2}^{2} d t+\int_{0}^{T}\left(\gamma_{2}|u(t)|_{m}^{2}+\gamma_{3}\left|u^{\prime}(t)\right|_{m}^{2}\right) d t\right)$ on all $(X, u) \in L_{\mathrm{ad}}^{2}\left(0, T ; L^{2}\left(\Omega ; L^{2}\right)\right) \times \mathcal{U}_{\mathrm{ad}}$ subject to $(1.1)$.

Here, $u^{\prime}$ is the time derivative in the sense of distributions $[\Phi(u)=\infty$ if there is no such derivative], $\gamma_{j} \geq 0,1 \leq j \leq 3, \mathbb{X}_{T} \in L^{2}\left(\Omega, \mathcal{F}_{T}, \mathbb{P} ; L^{2}\right)$ and $\mathbb{X}_{1} \in$ $L_{\text {ad }}^{2}\left(0, T ; L^{2}\left(\Omega ; L^{2}\right)\right)$ are given. In most situations, $\mathbb{X}_{1}$ is a given trajectory of the uncontrolled system or, in particular, a steady state solution.

As seen earlier, due to the conservation of $|X(t)|_{2}^{2}$, by normalizing the initial state we have $|X(t)|_{2}=1$, and so Problem $(\mathrm{P})$ reduces to

$$
\begin{aligned}
\underset{(u, X)}{\operatorname{Min}}\{ & -2 \mathbb{E} \operatorname{Re}\left(X(T), \mathbb{X}_{T}\right\rangle_{2}-2 \gamma_{1} \int_{0}^{T} \operatorname{Re}\left\langle X(t), \mathbb{X}_{1}(t)\right\rangle_{2} d t \\
& \left.+\int_{0}^{T}\left(\gamma_{2}|u(t)|_{m}^{2}+\gamma_{3}\left|u^{\prime}(t)\right|_{m}^{2}\right) d t .\right\}
\end{aligned}
$$

It should be said that in the quantum model $V$ is a given potential which describes the spatial profile of an external field, while the control input $u=\left\{u_{j}\right\}_{j=1}^{m}$ is its intensity. The objective of the control process is to steer the quantum system from an initial state $x$ to a target state $\mathbb{X}_{T}$ and also into the neighborhood of a given trajectory $\mathbb{X}_{1}$. The last term in the cost functional is the energy cost to obtain the desired objective.

Taking into account that in quantum mechanics the wave function $X$ is not a physical observable, a more realistic situation is where in the cost functional $\left|X(T)-\mathbb{X}_{T}\right|_{2}^{2}$ is replaced by $\left\langle Q(X(T))-\mathbb{X}_{T}, X(T)-\mathbb{X}_{T}\right\rangle_{2}$, where $Q$ is a selfadjoint operator in $L^{2}$. However, its treatment is essentially the same.

By $\Phi: L_{\text {ad }}^{2}\left(0, T ; \mathbb{R}^{m}\right) \rightarrow \mathbb{R}$, we denote the objective functional

$$
\begin{aligned}
\Phi(u)= & \mathbb{E}\left|X^{u}(T)-\mathbb{X}_{T}\right|_{2}^{2}+\gamma_{1} \mathbb{E} \int_{0}^{T}\left|X^{u}(t)-\mathbb{X}_{1}(t)\right|_{2}^{2} d t \\
& +\gamma_{2} \mathbb{E} \int_{0}^{T}|u(t)|_{m}^{2} d t+\gamma_{3} \mathbb{E} \int_{0}^{T}\left|u^{\prime}(t)\right|_{m}^{2} d t
\end{aligned}
$$

we may reformulate Problem $(\mathrm{P})$ as

(P) $\operatorname{Min}\left\{\Phi(u) ; u \in \mathcal{U}_{\mathrm{ad}}, X^{u}\right.$ satisfies (1.1) $\}$.

It should be said that, since Problem $(\mathrm{P})$ is a nonconvex minimization problem, in general it is not well-posed. However, if $\gamma_{2}, \gamma_{3}=0$, we have the following generic existence result.

Proposition 2.3. Assume Hypothesis (H0), and $\gamma_{2}=\gamma_{3}=0$. Then there is a residual set

$$
\mathcal{G} \subset L^{2}\left(\Omega, \mathcal{F}_{T}, \mathbb{P}, L^{2}\right) \times L_{\text {ad }}^{2}\left(0, T ; L^{2}\left(\Omega ; L^{2}\right)\right)
$$


such that, for every $\left(\mathbb{X}_{T}, \mathbb{X}_{1}\right) \in \mathcal{G}$, Problem (P) has at least one solution $u \in$ $\mathcal{U}_{\mathrm{ad}}$.

This is an immediate consequence of a well-known result of Edelstein [11] on existence of nearest points of closed sets in uniformly convex Banach spaces. Indeed, if we set $\mathcal{Y}=\left\{Y=\left(X^{u}(T), X^{u}\right) ; u \in \mathcal{U}_{\mathrm{ad}}\right\}$, it follows that $\mathcal{Y}$ is a closed subset of $L^{2}\left(\Omega ; \mathcal{F}_{T}, \mathbb{P}, L^{2}\right) \times L_{\text {ad }}^{2}\left(0, T ; L^{2}\left(\Omega ; L^{2}\right)\right.$ ) (see, e.g., the proof of Lemmas 3.2 and 3.3) and so, rewriting Problem (P) as

$$
\operatorname{Min}\left\{\left\|\left(\mathbb{X}_{T}, \mathbb{X}_{1}\right)-Y\right\|_{*}^{2} ; Y \in \mathcal{Y}\right\},
$$

where $\|\cdot\|_{*}$ is the norm of $L^{2}\left(\Omega ; \mathcal{F}_{T}, \mathbb{P}, L^{2}\right) \times L_{\text {ad }}^{2}\left(0, T ; L^{2}\left(\Omega ; L^{2}\right)\right)$, we arrive at the desired conclusion.

However, for the general cases $\gamma_{2}, \gamma_{3} \neq 0$, the existence of a solution in Problem $(\mathrm{P})$ does not follow by standard compactness techniques used in deterministic optimization problems. The main reason is that, even if a space $\mathcal{Y}$ is compactly imbedded into another space $\mathcal{Z}$, one generally does not have the compact imbedding from $L^{p}(\Omega ; \mathcal{Y})$ into $L^{p}(\Omega ; \mathcal{Z}), 1 \leq p \leq \infty$. Here, we consider the existence for relaxed versions of Problem $(\mathrm{P})$ to be defined below.

Definition 2.4. Let $\mathcal{Y}:=L^{2}\left(\mathbb{R}^{d}\right) \times L^{2}\left((0, T) \times \mathbb{R}^{d}\right) \times C\left([0, T] ; \mathbb{R}^{N}\right) \times$ $L^{2}\left(0, T ; \mathbb{R}^{m}\right) \times L^{2}\left((0, T) \times \mathbb{R}^{d}\right)$ and $\left(\Omega^{*}, \mathcal{F}^{*},\left(\mathcal{F}_{t}^{*}\right)_{t \geq 0}\right)$ be a new filtered probability space, carrying $\left(\mathbb{X}_{T}^{*}, \mathbb{X}_{1}^{*}, \beta^{*}, u^{*}, X^{*}\right)$ in $\mathcal{Y}$. Define $L_{\mathrm{ad}^{*}}^{2}\left(0, T ; L^{2}\left(\Omega ; L^{2}\right)\right)$, $\mathcal{U}_{\text {ad* }}$ and $\Phi^{*}\left(u^{*}\right)$ similarly as above on this new filtered probability space.

The system $\left(\Omega^{*}, \mathcal{F}^{*}, \mathbb{P}^{*},\left(\mathcal{F}_{t}^{*}\right)_{t \geq 0}, \beta^{*}, u^{*}, X^{*}\right)$ is said to be admissible, if $\mathbb{X}_{T}^{*} \in L^{2}\left(\Omega, \mathcal{F}_{T}^{*}, \mathbb{P}^{*} ; L^{2}\right), \mathbb{X}_{1}^{*} \in L_{\text {ad }}^{2}\left(0, T ; L^{2}\left(\Omega ; L^{2}\right)\right), \beta^{*}=\left(\beta_{1}^{*}, \ldots, \beta_{N}^{*}\right)$ is an $\left(\mathcal{F}_{t}^{*}\right)_{t \geq 0}$-adapted $\mathbb{R}^{N}$-valued Wiener process, the joint distributions of $\left(\mathbb{X}_{T}^{*}, \mathbb{X}_{1}^{*}\right.$, $\left.\beta^{*}\right)$ and $\left(\mathbb{X}_{T}, \mathbb{X}_{1}, \beta\right)$ coincide, $u^{*} \in \mathcal{U}_{\mathrm{ad}}{ }^{*}$ and $X^{*}$ is an $\left(\mathcal{F}_{t}^{*}\right)_{t \geq 0}$-adapted $L^{2}$-valued process that satisfies equation (1.1) corresponding to $\left(\beta^{*}, u^{*}\right)$.

The admissible system $\left(\Omega^{*}, \mathcal{F}^{*}, \mathbb{P}^{*},\left(\mathcal{F}_{t}^{*}\right)_{t \geq 0}, \beta^{*}, u^{*}, X^{*}\right)$ is said to be a relaxed solution to the optimal control problem $(\mathrm{P})$, if

$$
\Phi^{*}\left(u^{*}\right) \leq \inf \left\{\Phi(u) ; u \in \mathcal{U}_{\mathrm{ad}}, X^{u} \text { satisfies }(1.1)\right\} .
$$

Similar to martingale and strong solutions, one can have a nonrelaxed optimal control by using, for example, the Gyöngy-Krylov argument as in [15], as long as the uniqueness of the optimal control is obtained, which reduces to the uniqueness of solutions to a forward-backward equation [see (1.1) and (2.9) below with $u$ replaced by (2.12)] and which is generally very difficult due to the nonconvexity of the optimal control problem (P).

We first prove that, under the regular condition of controls (i.e., $\gamma_{3}>0$ ), there exists a relaxed solutions for the exponents of the nonlinearity in exactly the masssubcritical range. A similar problem was studied in [16] (see also [6, 19]) in the deterministic case. We have the following. 
THEOREM 2.5. Consider $\Phi$ with $\gamma_{3}>0$. Assume (H0). Then, for each $x \in L^{2}$, $0<T<\infty$, there exists at least one relaxed solution in the sense of Definition 2.4 to the optimal problem $(\mathrm{P})$.

The proof is mainly based on the Skorohod representation theorem and pathwise analysis of solutions by the rescaling approach devoloped in [1]. We would also like to mention that the rescaling approach allows to obtain pathwise continuous dependence of solutions on controls.

In order to construct a relaxed solution with equality in (2.8) in the more difficult irregular case (i.e., $\gamma_{3}=0$ ), we will employ the Ekeland principle and work with the dual backward stochastic equation below:

$$
\begin{aligned}
d Y= & -i \Delta Y d t-\lambda i h_{1}\left(X^{u}\right) Y d t+\lambda i h_{2}\left(X^{u}\right) \bar{Y} d t \\
& +\mu Y d t-i V_{0} Y d t-i u \cdot V Y d t \\
& +\gamma_{1}\left(X^{u}-\mathbb{X}_{1}\right) d t-\sum_{k=1}^{N} \overline{\mu_{k}} e_{k} Z_{k} d t+\sum_{k=1}^{N} Z_{k} d \beta_{k}(t), \\
Y(T)= & -\left(X^{u}(T)-\mathbb{X}_{T}\right),
\end{aligned}
$$

where

$$
h_{1}\left(X^{u}\right):=\frac{\alpha+1}{2}\left|X^{u}\right|^{\alpha-1}, \quad h_{2}\left(X^{u}\right):=\frac{\alpha-1}{2}\left|X^{u}\right|^{\alpha-3}\left(X^{u}\right)^{2} .
$$

The functions $h_{j}, j=1,2$, are the complex derivatives of the complex function $z \rightarrow|z|^{\alpha-1} z$, that is, $h_{1}(z)=\partial_{z}\left(|z|^{\alpha-1} z\right)$ and $h_{2}(z)=\partial_{\bar{z}}\left(|z|^{\alpha-1} z\right), z \in \mathbb{C}$.

However, the singular coefficient $h_{2}\left(X^{u}\right)$ in (2.9) and the weak regularity effect of the Schrödinger group make it quite difficult to obtain the existence and integrability of the backward solution. The standard method to derive a global estimate for $\mathbb{E}\|Y\|_{C\left([0, T] ; L^{2}\right)}^{2}$ from the Itô formula applied to $|Y(t)|_{2}^{2}$ are not applicable in the nonlinear case.

The idea here is to apply duality analysis to reduce the analysis of the backward stochastic equation to that of the dual equation (4.26) below [see also the equation of variation (4.3) below]. By virtue of the forward character of the dual equation, we will apply the rescaling approach and the Strichartz estimates, instead of the Itô formula for $|Y(t)|_{2}^{2}$, to control the singular coefficient $h_{2}\left(X^{u}\right)$ and to obtain pathwise estimates of solutions on small intervals, which then by iteration yield the global pathwise estimates (4.10), (4.27) below. To this aim, we consider in this case the following basic hypothesis:

(H2) $2 \leq \alpha<1+\frac{4}{d}, 1 \leq d \leq 3$, and $e_{k}$ are constants, $1 \leq k \leq N$.

[In the case where $e_{k}$ are not constant, which is ruled out here, there arise some delicate problems related to the nonintegrability of $\left(B_{k}\right)^{c\left(B_{k}\right)^{2 / \theta}}$, where $B_{k}:=\sup _{t \in[0, T]}\left|\beta_{k}(t)\right|, \theta \in(0,1)$ and $c>0,1 \leq k \leq N$.] 
It is easily seen that (H2) implies (H0) and (H1) and also that (H2) is fulfilled in some important physical models, for instance, the Gross-Pitaevskii model when $d=1$ [16]. As a matter of fact, under Hypothesis (H2), one has not only (2.8) with equality, but also that the optimal pair $(X, u)$ satisfies the stochastic maximum principle. The main result is formulated below.

TheOREM 2.6. Consider $\Phi$ with $\gamma_{3}=0$. Assume Hypothesis $(\mathrm{H} 2)$, and $\mathbb{X}_{T} \in$ $L^{2+v}\left(\Omega ; H^{1}\right), \mathbb{X}_{1} \in L^{2+v}\left(\Omega ; L^{2}\left(0, T ; H^{1}\right)\right)$ for some small $v \in(0,1)$.

Then, for each $x \in H^{1}, 0<T<\infty$, there exists a relaxed solution $\left(\Omega^{*}, \mathscr{F}^{*}\right.$, $\left.\mathbb{P}^{*},\left(\mathscr{F}_{t}^{*}\right)_{t \geq 0}, \beta^{*}, u^{*}, X^{*}\right)$ in the sense of Definition 2.6 to Problem $(\mathrm{P})$, such that

$$
\Phi^{*}\left(u^{*}\right)=\inf \left\{\Phi(u) ; u \in \mathcal{U}_{\mathrm{ad}}, X^{u} \text { satisfies }(1.1)\right\} .
$$

Moreover, we have (the stochastic maximum principle)

$$
u^{*}(t)=P_{U}\left(\frac{1}{\gamma_{2}} \operatorname{Im} \int_{\mathbb{R}^{d}} V(\xi) X^{*}(t, \xi) \overline{Y^{*}}(t, \xi) d \xi\right) \quad \forall t \in[0, T], \mathbb{P}^{*} \text {-a.s. }
$$

where $P_{U}$ is the projection on $U$, and $\left(Y^{*}, Z^{*}\right)$ is the solution to the dual backward stochastic equation (2.9) with $\mathbb{X}_{T}, \mathbb{X}_{1}, \beta, u, X^{u}$ replaced by $\mathbb{X}_{T}^{*}, \mathbb{X}_{1}^{*}, \beta^{*}, u^{*}, X^{*}$, respectively.

In the case where $\mu_{k}=0,1 \leq k \leq N$, the noise $W$ in (1.1) disappears and so, (1.1) is the deterministic controlled nonlinear Schrödinger equation. In this case, for the initial datum $x \in H^{1}$, the optimal control indeed exists for the exponent $\alpha \geq 2$ in the energy-subcritical case (H1), which is also new in the literature.

THEOREM 2.7. In the deterministic case (i.e., $\mu_{k}=0,1 \leq k \leq N$ ), consider $\Phi$ in (2.6) with $\gamma_{3}=0$ and the exponent $\alpha \geq 2$ in the range specified in Hypothesis (H1). Assume that $\mathbb{X}_{T} \in H^{1}$ and $\mathbb{X}_{1} \in L^{2}\left(0, T ; H^{1}\right)$.

Then, for each $x \in H^{1}, 0<T<\infty$, there exists an optimal control u to Problem (P) such that

$$
\Phi(u)=\inf \left\{\Phi(v) ; v \in \mathcal{U}_{\mathrm{ad}}, X^{v} \text { satisfies }(2.1)\right\}
$$

Moreover,

$$
u(t)=P_{U}\left(\frac{1}{\gamma_{2}} \operatorname{Im} \int_{\mathbb{R}^{d}} V(\xi) X(t, \xi) \bar{Y}(t, \xi) d \xi\right) \quad \forall t \in[0, T],
$$

where $P_{U}$ is the projection on $U$, and $Y$ is the solution to the backward equation (2.9) with $Z=0$.

REMARK 2.8. Optimal bilinear control is also studied in [18] and [16] for linear and nonlinear deterministic Schrödinger equations, respectively. In both papers, some compactness conditions of initial data or controls are needed for the 
existence of the optimal control. More precisely, in [18] the initial data belong to a compact subspace of $L^{2}$, while in [16] the minimizing controls are bounded in $H^{1}[0, T]$, hence compact in $L^{2}[0, T]$. In contrast to this, in Theorem 2.7 the existence of the optimal control is obtained without these conditions, and unlike in [16], less regularity of the initial data is required for the maximum principle (2.14). The proof is quite different; it applies to more general initial data and as well to the stochastic case. Moreover, taking into account the importance of the energy state space $H^{1}$, we also expect the results and methods developed here to be applied to the study of blow-up and scattering phenomena (see, e.g., $[3,20])$.

REMARK 2.9. For the control in the diffusion coefficient, it is technically more complicated to treat the equation of variation and the corresponding stochastic backward equation. It also involves the analysis of a second-order adjoint equation in the stochastic maximum principle (see, e.g., Chapter 3 in [30]). Including control in the diffusion coefficient will be one subject that we plan to analyze in future.

The proof of Theorem 2.7 follows the lines of that of Theorem 2.6, the main part of which are the analysis of equation of variation (4.3) as well as of the backward stochastic equation (2.9), and the tightness of controls.

The key idea to obtain the tightness of controls in this irregular case is to employ the Ekeland principle, as well as the directional derivative of $\Phi$, to obtain the representation formula of the minimizing controls [see (5.10) below]. Then, by virtue of the integrability of the forward and backward solutions to (1.1) and (2.9), respectively, one is able to obtain the tightness of controls in the space $L^{1}\left(0, T ; \mathbb{R}^{d}\right)$, which consequently yields equality in (2.8) by analogous arguments as in the proof of Theorem 2.5.

As mentioned above, the proof of integrability of the stochastic backward solution relies on duality analysis, which is also of independent interest.

The remaining part of this paper is organized as follows. Section 3 includes the proof of Theorem 2.5. Section 4 and Section 5 are mainly devoted to the proof of Theorem 2.6. Section 4 is concerned with the directional derivative of $\Phi$, which requires the analysis of the equation of variation (4.3) and of the backward stochastic equation (2.9). Section 5 mainly contains the proof for the tightness of controls. The proof of Theorem 2.7 is also included there. For simplicity of the exposition, some auxiliary lemmas and technical proofs are postponed to the Appendix.

Notation. For $1 \leq p \leq \infty$, we denote by $L^{p}\left(\mathbb{R}^{d}\right)=L^{p}$ the space of all Lebesgue $p$-integrable (complex-valued) functions on the real Euclidean space $\mathbb{R}^{d}$. The norm of $L^{p}$ is denoted by $|\cdot|_{L^{p}}$, and $p^{\prime} \in[1, \infty]$ denotes the unique number such that $\frac{1}{p}+\frac{1}{p^{\prime}}=1$. In particular, the Hilbert space $L^{2}\left(\mathbb{R}^{d}\right)$ is endowed with the scalar product

$$
\langle y, z\rangle_{2}=\int_{\mathbb{R}^{d}} y(\xi) \bar{z}(\xi) d \xi ; \quad y, z \in L^{2}\left(\mathbb{R}^{d}\right)
$$


where $\bar{z}$ is the complex conjugate of $z \in \mathbb{C}$. We also use $|\cdot|_{2}=|\cdot|_{L^{2}} \cdot W^{1, p}=$ $W^{1, p}\left(\mathbb{R}^{d}\right)$ is the classical Sobolev space $\left\{v \in L^{p} ; \nabla v \in L^{p}\right\}$ with the norm $\|v\|_{W^{1, p}}=|v|_{L^{p}}+|\nabla v|_{L^{p}}, H^{1}=W^{1,2}$ and $H^{-1}$ is the dual space of $H^{1}$.

By $L^{q}\left(0, T ; L^{p}\right)$, we denote the space of all integrable $L^{p}$-valued functions $u:(0, T) \rightarrow L^{p}$ with the norm

$$
\|u\|_{L^{q}\left(0, T ; L^{p}\right)}=\left(\int_{0}^{T}\left(\int_{\mathbb{R}^{d}}|u(t, \xi)|^{p} d \xi\right)^{\frac{q}{p}} d t\right)^{\frac{1}{q}} .
$$

By $C\left([0, T] ; L^{p}\right)$, we denote the standard space of all $L^{p}$-valued continuous functions on $[0, T]$ with the sup norm in $t . L^{q}\left(0, T ; W^{1, p}\right)$ and $C\left([0, T] ; H^{1}\right)$ are defined similarly. $\mathcal{D}\left(0, T ; \mathbb{R}^{m}\right)$ is the set of all $\mathbb{R}^{m}$-valued smooth and compactly supported functions, and $\mathcal{D}^{\prime}\left(0, T ; \mathbb{R}^{m}\right)$ is its dual space.

Let $|\cdot|_{m}$ denote the Euclidean norm in $\mathbb{R}^{m}$ and $u \cdot v$ the scalar product of vectors $u, v \in \mathbb{R}^{m}$. We shall use standard notation to represent spaces of infinite dimensional stochastic processes (see, e.g., $[8,21]$ ).

Throughout this paper, we use $C$ for various constants that may change from line to line.

3. Proof of Theorem 2.5. We set $I:=\inf \left\{\Phi(u) ; u \in \mathcal{U}_{\mathrm{ad}}, X^{u}\right.$ satisfies (1.1) $\}>0$ and consider a sequence $\left\{u_{n}\right\} \subset \mathcal{U}_{\text {ad }}$ such that

$$
I \leq \Phi\left(u_{n}\right) \leq I+n^{-1} \quad \forall n \in \mathbb{N} .
$$

Since $\gamma_{3}>0$, this yields

$$
\sup _{n \geq 1} \mathbb{E} \int_{0}^{T}\left(\left|u_{n}(t)\right|_{m}^{2}+\left|u_{n}^{\prime}(t)\right|_{m}^{2}\right) d t<\infty
$$

LEMMA 3.1. Let $\mathbb{P} \circ u_{n}^{-1}$ be the laws of $u_{n}, n \geq 1$. Then $\left\{\mathbb{P} \circ u_{n}^{-1}\right\}$ is tight on $C\left([0, T] ; \mathbb{R}^{m}\right)$.

PROOF. By the Arzelà theorem, it suffices to show that

$$
\begin{aligned}
& \lim _{R \rightarrow \infty} \sup _{n \geq 1} \mathbb{P} \circ u_{n}^{-1}\left\{v \in C\left([0, T] ; \mathbb{R}^{m}\right): \sup _{t \in[0, T]}|v(t)|_{m}>R\right\} \\
& \quad=0
\end{aligned}
$$

and for any $\varepsilon>0$,

$$
\begin{aligned}
& \lim _{\delta \rightarrow 0} \sup _{n \geq 1} \mathbb{P} \circ u_{n}^{-1}\left\{v \in C\left([0, T] ; \mathbb{R}^{m}\right): \sup _{|t-s| \leq \delta}|v(t)-v(s)|_{m}>\varepsilon\right\} \\
& \quad=0 .
\end{aligned}
$$


In fact, (3.3) follows immediately form the uniform boundedness of $\left\{u_{n}\right\}$, while (3.4) follows by (3.2) and

$$
\begin{aligned}
\sup _{n \geq 1} \mathbb{P} & \circ u_{n}^{-1}\left\{v \in C\left([0, T] ; \mathbb{R}^{m}\right): \sup _{|t-s| \leq \delta}|v(t)-v(s)|_{m}>\varepsilon\right\} \\
& \leq \frac{1}{\varepsilon} \sup _{n \geq 1} \mathbb{E} \sup _{|t-s| \leq \delta}\left|u_{n}(t)-u_{n}(s)\right|_{m} \\
& \leq \frac{\delta^{\frac{1}{2}}}{\varepsilon} \sup _{n \geq 1} \mathbb{E}\left\|u_{n}^{\prime}\right\|_{L^{2}\left(0, T ; \mathbb{R}^{m}\right)} \rightarrow 0 \quad \text { as } \delta \rightarrow 0 .
\end{aligned}
$$

Now, consider the sequence $\mathcal{X}_{n}:=\left(\mathbb{X}_{T}, \mathbb{X}_{1}, \beta, u_{n}\right)$ with $\beta=\left(\beta_{1}, \ldots, \beta_{N}\right)$ in the space $\mathcal{Y}:=L^{2}\left(\mathbb{R}^{d}\right) \times L^{2}\left((0, T) \times \mathbb{R}^{d}\right) \times C\left([0, T] ; \mathbb{R}^{N}\right) \times C\left([0, T] ; \mathbb{R}^{m}\right)$. Lemma 3.1 implies that the laws of $\mathcal{X}_{n}, n \in \mathbb{N}$, are tight in the space $\mathcal{Y}$. Then, by Prohorov's theorem, they are weakly compact and so, by the Skorohod representation theorem, there exist a probability space $\left(\Omega^{*}, \mathcal{F}^{*}, \mathbb{P}^{*}\right)$ and $\mathcal{X}_{n}^{*}:=$ $\left(\left(\mathbb{X}_{T}^{*}\right)_{n}, \mathbb{X}_{1, n}^{*}, \beta_{n}^{*}, u_{n}^{*}\right), \mathcal{X}^{*}:=\left(\mathbb{X}_{T}^{*}, \mathbb{X}_{1}^{*}, \beta^{*}, u^{*}\right)$ in $\mathcal{Y}, n \in \mathbb{N}$, such that the joint distribution of $\mathcal{X}_{n}^{*}$ and $\mathcal{X}_{n}$ coincide, and $\mathbb{P}^{*}$-a.s., as $n \rightarrow \infty$,

$$
\begin{aligned}
\beta_{n}^{*} \rightarrow \beta^{*} & \text { in } C\left([0, T] ; \mathbb{R}^{N}\right), \\
\left(\mathbb{X}_{T}^{*}\right)_{n} \rightarrow \mathbb{X}_{T}^{*} & \text { in } L^{2}\left(\mathbb{R}^{d}\right), \\
\mathbb{X}_{1, n}^{*} \rightarrow \mathbb{X}_{1}^{*} & \text { in } L^{2}\left((0, T) \times \mathbb{R}^{d}\right)
\end{aligned}
$$

and

$$
u_{n}^{*} \rightarrow u^{*} \quad \text { in } C\left([0, T] ; \mathbb{R}^{m}\right) .
$$

Note that, $\left(\left(\mathbb{X}_{T}^{*}\right)_{n}, \mathbb{X}_{1, n}^{*}, \beta_{n}^{*}\right)$ has the same distribution as $\left(\mathbb{X}_{T}, \mathbb{X}_{1}, \beta\right)$, and so does the limit $\left(\mathbb{X}_{T}^{*}, \mathbb{X}_{1}^{*}, \beta^{*}\right)$.

For each $n \geq 1$, define $\mathcal{F}_{t, n}^{*}:=\sigma\left(\mathcal{X}_{n}^{*}(s), s \leq t\right)$. Then $\mathbb{X}_{n}^{*}(T) \in L^{2}\left(\Omega, \mathcal{F}_{T}^{*}\right.$,

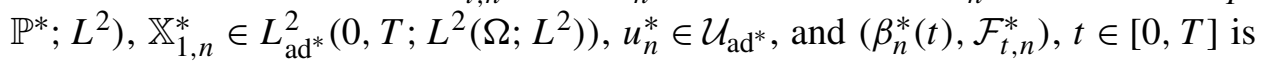
a Wiener process. It follows from Proposition 2.2 that, under the hypothesis (H0), for each $\left(\beta_{n}^{*}, u_{n}^{*}\right)$ there exists a unique strong $L^{2}$-solution $X_{n}^{*}$ to (1.1). Hence, $\left(\Omega^{*}, \mathcal{F}^{*}, \mathbb{P}^{*},\left(\mathcal{F}_{t}^{*}\right)_{t \geq 0}, \beta_{n}^{*}, u_{n}^{*}, X_{n}^{*}\right)$ is an admissible system.

Moreover, since the solution to (1.1) is a measurable map of Brownian motions and controls, we also have that the distribution of $\left(\left(\mathbb{X}_{T}^{*}\right)_{n}, \mathbb{X}_{1, n}^{*}, \beta_{n}^{*}, u_{n}^{*}, X_{n}^{*}\right)$ is the same as that of $\left(\mathbb{X}_{T}, \mathbb{X}_{1}, \beta, u_{n}, X_{n}\right)$, where $X_{n}$ is the solution to (1.1) corresponding to $\left(\beta, u_{n}\right)$. In particular, $\Phi^{*}\left(u_{n}^{*}\right)=\Phi\left(u_{n}\right)$.

Similarly, set $\mathcal{F}_{t}^{*}:=\sigma\left(\mathcal{X}^{*}(s), s \leq t\right)$ and let $X^{*}$ be the unique strong $L^{2}$ solution to $(1.1)$ corresponding to $\left(\beta^{*}, u^{*}\right)$. Then $\left(\Omega^{*}, \mathcal{F}^{*}, \mathbb{P}^{*},\left(\mathcal{F}_{t}^{*}\right)_{t \geq 0}, \beta^{*}, u^{*}\right.$, $\left.X^{*}\right)$ is an admissible system.

Below, we consider the derivatives of $u_{n}^{*}$ and $u^{*}$. For each $n \geq 1$, define $\left(u_{n}^{*}\right)^{\prime} \in \mathcal{D}^{\prime}\left(0, T ; \mathbb{R}^{m}\right)$ in the distribution sense, that is, $\left(\left(u_{n}^{*}\right)^{\prime}, v\right)=-\left(u_{n}^{*}, v^{\prime}\right), \forall v \in$ 
$\mathcal{D}\left(0, T ; \mathbb{R}^{m}\right)$, where $\left(\right.$, ) denotes the pairing between $\mathcal{D}^{\prime}\left(0, T ; \mathbb{R}^{m}\right)$ and $\mathcal{D}(0, T$; $\left.\mathbb{R}^{m}\right)$. We claim that $\left(u_{n}^{*}\right)^{\prime}$ has the same distribution as $u_{n}^{\prime}$ in $\mathcal{D}^{\prime}\left(0, T ; \mathbb{R}^{m}\right)$, and $\mathbb{E}^{*}\left\|\left(u_{n}^{*}\right)^{\prime}\right\|_{L^{2}\left(0, T ; \mathbb{R}^{m}\right)}^{2}=\mathbb{E}\left\|u_{n}^{\prime}\right\|_{L^{2}\left(0, T ; \mathbb{R}^{m}\right)}^{2}$. It follows from (3.2) that there exists $v^{*} \in L^{2}\left(\Omega^{*} ; L^{2}\left(0, T ; \mathbb{R}^{m}\right)\right)$, such that

$$
\left(u_{n}^{*}\right)^{\prime} \rightarrow v^{*} \quad \text { weakly in } L^{2}\left(\Omega^{*} ; L^{2}\left(0, T ; \mathbb{R}^{m}\right)\right), n \rightarrow \infty .
$$

Indeed, for any $l \geq 1, v_{j} \in \mathcal{D}\left(0, T ; \mathbb{R}^{m}\right), 1 \leq j \leq l$, and $c \in \mathbb{R}^{l}$,

$$
\begin{aligned}
\mathbb{E}^{*} \exp \left(i \sum_{j=1}^{l} c_{j}\left(\left(u_{n}^{*}\right)^{\prime}, v_{j}\right)\right) & =\mathbb{E}^{*} \exp \left(-i\left(u_{n}^{*}, \sum_{j=1}^{l} c_{j} v_{j}^{\prime}\right)\right) \\
& =\mathbb{E} \exp \left(-i\left(u_{n}, \sum_{j=1}^{l} c_{j} v_{j}^{\prime}\right)\right) \\
& =\mathbb{E} \exp \left(i \sum_{j=1}^{l} c_{j}\left(u_{n}^{\prime}, v_{j}\right)\right),
\end{aligned}
$$

which implies that the distributions of $\left(u_{n}^{*}\right)^{\prime}$ and $u_{n}^{\prime}$ coincide. Moreover, if $\mathscr{D}:=$ $\left\{v_{n}\right\}$ is a dense subset in $\mathcal{D}\left(0, T ; \mathbb{R}^{m}\right)$ [hence also dense in $L^{2}\left(0, T ; \mathbb{R}^{m}\right)$ ], we have

$$
\mathbb{E}^{*} \sup _{n \geq 1} \frac{\left|\left(\left(u_{n}^{*}\right)^{\prime}, v_{n}\right)\right|^{2}}{\left\|v_{n}\right\|_{L^{2}\left(0, T ; \mathbb{R}^{m}\right)}^{2}}=\mathbb{E} \sup _{n \geq 1} \frac{\left|\left(u_{n}^{\prime}, v_{n}\right)\right|^{2}}{\left\|v_{n}\right\|_{L^{2}\left(0, T ; \mathbb{R}^{m}\right)}^{2}}=\mathbb{E}\left\|u_{n}^{\prime}\right\|_{L^{2}\left(0, T ; \mathbb{R}^{m}\right)}^{2}<\infty,
$$

which implies that $\mathbb{E}^{*}\left\|\left(u_{n}^{*}\right)^{\prime}\right\|_{L^{2}\left(0, T ; \mathbb{R}^{m}\right)}^{2}=\mathbb{E}\left\|u_{n}^{\prime}\right\|_{L^{2}\left(0, T ; \mathbb{R}^{m}\right)}^{2}$, as claimed.

Similarly, define $\left(u^{*}\right)^{\prime} \in \mathcal{D}^{\prime}\left(0, T ; \mathbb{R}^{m}\right)$ in the distribution sense. We have

$$
\left(u^{*}\right)^{\prime}=v^{*} \quad \text { in } L^{2}\left(0, T ; \mathbb{R}^{m}\right), \mathbb{P}^{*} \text {-a.s. }
$$

Indeed, for any $v \in \mathscr{D}$ and $\psi \in L^{\infty}\left(\Omega^{*}\right)$, by (3.8), the dominated convergence theorem and the weak convergence (3.9), it follows that

$$
\begin{aligned}
\mathbb{E}^{*}\left(\left(u^{*}\right)^{\prime}, v\right) \psi & =-\mathbb{E}^{*} \psi\left(\int_{0}^{T} u^{*}(t) \cdot v^{\prime}(t) d t\right) \\
& =-\lim _{n \rightarrow \infty} \mathbb{E}^{*} \psi\left(\int_{0}^{T} u_{n}^{*}(t) \cdot v^{\prime}(t) d t\right) \\
& =\lim _{n \rightarrow \infty} \mathbb{E}^{*} \int_{0}^{T}\left(u_{n}^{*}\right)^{\prime}(t) \cdot v(t) \psi d t \\
& =\mathbb{E}^{*} \int_{0}^{T} v^{*}(t) \cdot v(t) \psi d t=\mathbb{E}^{*}\left(v^{*}, v\right) \psi .
\end{aligned}
$$

Hence, $\left(\left(u^{*}\right)^{\prime}, v\right)=\left(v^{*}, v\right), \mathbb{P}^{*}$-a.s., $v \in \mathscr{D}$. Since $\mathscr{D}$ is countable and dense in $L^{2}\left(0, T ; \mathbb{R}^{m}\right),(3.10)$ follows.

Next, we show that the solutions to (1.1) depend pathwisely continuous on the controllers, by using the rescaling approach developed in $[1,2]$. 
LEMMA 3.2. Let $X_{n}^{*}$ (resp., $X^{*}$ ) be the solution to (1.1) corresponding to $\left(\beta_{n}^{*}, u_{n}^{*}\right)\left[\right.$ resp., $\left.\left(\beta^{*}, u^{*}\right)\right]$ as above, $n \geq 1$. Assume the conditions in Theorem 2.5 to hold. Then, for each $x \in L^{2}, 0<T<\infty$ and each Strichartz pair $(p, q)$, we have $\mathbb{P}^{*}$-a.s., as $n \rightarrow \infty$,

$$
\left\|X_{n}^{*}-X^{*}\right\|_{C\left([0, T] ; L^{2}\right)}+\left\|X_{n}^{*}-X^{*}\right\|_{L^{q}\left(0, T ; L^{p}\right)} \rightarrow 0 .
$$

PROOF. Set $W_{n}^{*}(t, \xi)=\sum_{j=1}^{N} \mu_{j} e_{j}(\xi) \beta_{j, n}^{*}(t), W^{*}(t, \xi)=\sum_{j=1}^{N} \mu_{j} e_{j}(\xi) \times$ $\beta_{j}^{*}(t), t \geq 0, \xi \in \mathbb{R}^{d}$. We may assume $T \geq 1$ without loss of generality.

Note that, in the conservative case,

$$
\left|X_{n}^{*}(t)\right|_{2}=|x|_{2}<\infty, \quad t \in[0, T], n \geq 1, \mathbb{P}^{*} \text {-a.s. }
$$

Using the rescaling transformation,

$$
y_{n}^{*}=e^{-W_{n}^{*}} X_{n}^{*},
$$

we deduce from (1.1) with $X, u, \beta_{j}$ replaced by $X_{n}^{*}, u_{n}^{*}$ and $\beta_{j, n}^{*}$, respectively, that

$$
\begin{aligned}
d y_{n}^{*} & =A_{n}^{*}(t) y_{n}^{*} d t-\lambda i\left|y_{n}^{*}\right|^{\alpha-1} y_{n}^{*} d t+f\left(u_{n}^{*}\right) y_{n}^{*} d t, \\
y_{n}^{*}(0) & =x,
\end{aligned}
$$

where $A_{n}^{*}(t)=-i\left(\Delta+b_{n}^{*}(t) \cdot \nabla+c_{n}^{*}(t)\right), \quad b_{n}^{*}(t)=2 \nabla W_{n}^{*}(t), \quad c_{n}^{*}(t)=$ $\sum_{j=1}^{d}\left(\partial_{j} W_{n}^{*}(t)\right)^{2}+\Delta W_{n}^{*}(t)$, and $f\left(u_{n}^{*}\right)=-i\left(V_{0}+u_{n}^{*} \cdot V\right)$.

It suffices to prove that for each Strichartz pair $(p, q), \mathbb{P}^{*}$-a.s.,

$$
\left\|y_{n}^{*}-y^{*}\right\|_{L^{\infty}\left(0, T ; L^{2}\right)}+\left\|y_{n}^{*}-y^{*}\right\|_{L^{q}\left(0, T ; L^{p}\right)} \rightarrow 0 \quad \text { as } n \rightarrow \infty .
$$

Now, we will prove (3.15) for the Strichartz pair $(p, q)=\left(\alpha+1, \frac{4(\alpha+1)}{d(\alpha-1)}\right)$. The general case will follow immediately from the Strichartz estimates (see, e.g., [1], Lemma 4.1 or [2], (A.1)).

To this end, we prove first that

$$
\sup _{n \geq 1}\left\|y_{n}^{*}\right\|_{L^{q}\left(0, T ; L^{p}\right)}<\infty, \quad \mathbb{P}^{*} \text {-a.s. }
$$

Applying the Strichartz estimates to (3.14) yields

$$
\left\|y_{n}^{*}\right\|_{L^{q}\left(0, t ; L^{p}\right)} \leq C_{T}\left[|x|_{2}+\left\|\lambda i\left|y_{n}^{*}\right|^{\alpha-1} y_{n}^{*}\right\|_{L^{q^{\prime}}\left(0, t ; L^{p^{\prime}}\right)}+\left\|f\left(u_{n}^{*}\right) y_{n}^{*}\right\|_{L^{1}\left(0, t ; L^{2}\right)}\right] .
$$

[Note that, the Strichartz coefficient $C_{T}$ is independent of $n$, since by (3.6) $\sup _{n \geq 1}\left\|W_{n}^{*}\right\|_{C\left([0, T] ; L^{\infty}\right)}<\infty, \mathbb{P}^{*}$-a.s. $]$

By Hölder's inequality,

$$
\left\|\lambda i\left|y_{n}^{*}\right|^{\alpha-1} y_{n}^{*}\right\|_{L^{q^{\prime}}\left(0, t ; L^{p^{\prime}}\right)} \leq t^{\theta}\left\|y_{n}^{*}\right\|_{L^{q}\left(0, T ; L^{p}\right)}^{\alpha},
$$

where $\theta=1-\frac{d(\alpha-1)}{4}>0$, and by (3.12),

$$
\left\|f\left(u_{n}^{*}\right) y_{n}^{*}\right\|_{L^{1}\left(0, t ; L^{2}\right)} \leq T\left(\left|V_{0}\right|_{L^{\infty}}+D_{U}\|V\|_{L^{\infty}\left(\mathbb{R}^{d} ; \mathbb{R}^{m}\right)}\right)|x|_{2} .
$$


Thus,

$$
\left\|y_{n}^{*}\right\|_{L^{q}\left(0, t ; L^{p}\right)} \leq C_{T}\left(D(T)|x|_{2}+t^{\theta}\left\|y_{n}^{*}\right\|_{L^{q}\left(0, T ; L^{p}\right)}^{\alpha}\right),
$$

where $D(T):=1+T\left(\left|V_{0}\right|_{\infty}+D_{U}\|V\|_{L^{\infty}\left(0, T ; \mathbb{R}^{m}\right)}\right)$.

Choose $t \in[0, T]$ such that $C_{T} D(T)\left(|x|_{2}+1\right)=\left(1-\frac{1}{\alpha}\right)\left(\alpha C_{T} t^{\theta}\right)^{-\frac{1}{\alpha-1}}$, that is,

$$
t=\alpha^{-\frac{\alpha}{\theta}}(\alpha-1)^{\frac{\alpha-1}{\theta}}\left(|x|_{2}+1\right)^{-\frac{\alpha-1}{\theta}} C_{T}^{-\frac{\alpha}{\theta}} D(T)^{-\frac{\alpha-1}{\theta}}(\leq T) .
$$

Then, by Lemma A.1, we get

$$
\left\|y_{n}^{*}\right\|_{L^{q}\left(0, t ; L^{p}\right)} \leq \frac{\alpha}{\alpha-1} C_{T} D(T)|x|_{2} .
$$

Iterating similar estimates on $[j t,(j+1) t \wedge T], 1 \leq j \leq\left[\frac{T}{t}\right]$, we obtain

$$
\left\|y_{n}^{*}\right\|_{L^{q}\left(0, T ; L^{p}\right)} \leq\left(\left[\frac{T}{t}\right]+1\right)^{\frac{1}{q}} \frac{\alpha}{\alpha-1} C_{T} D(T)|x|_{2},
$$

which yields (3.16).

It remains to prove (3.15). Applying the Strichartz estimates to the equations of $y_{n}^{*}$ and $y^{*}$, we have for any $t \in(0, T)$,

$$
\begin{aligned}
\| y_{n}^{*}- & y^{*}\left\|_{L^{\infty}\left(0, t ; L^{2}\right)}+\right\| y_{n}^{*}-y^{*} \|_{L^{q}\left(0, t ; L^{p}\right)} \\
\leq & C_{T}\left[\left\|f\left(u_{n}^{*}\right) y_{n}^{*}-f\left(u^{*}\right) y^{*}\right\|_{L^{1}\left(0, t ; L^{2}\right)}\right. \\
& \left.+\left\|\left|y_{n}^{*}\right|^{\alpha-1} y_{n}^{*}-\left|y^{*}\right|^{\alpha-1} y^{*}\right\|_{L^{q^{\prime}\left(0, t ; L^{p^{\prime}}\right)}}\right]+w_{n}\left(y^{*}\right),
\end{aligned}
$$

where $w_{n}\left(y^{*}\right)=C_{T}\left(\left\|\left(b_{n}^{*}-b^{*}\right) \cdot \nabla y^{*}\right\|_{\tilde{X}_{[0, T]}^{\prime}}+\left\|\left(c_{n}^{*}-c^{*}\right) y^{*}\right\|_{L^{1}\left(0, T ; L^{2}\right)}\right)$, and $\tilde{X}_{[0, T]}^{\prime}$ is the dual space of the local smoothing space $\tilde{X}_{[0, T]}$ introduced in [22] up to time $T$ (see also the proof of Lemma 2.7 in [2]). Since $y^{*} \in \widetilde{X}_{[0, T]}$, by Proposition 2.3(a) in [22] and (3.6), we note that $\mathbb{P}^{*}$-a.s. as $n \rightarrow \infty$,

$$
w_{n}\left(y^{*}\right) \leq C_{T}\left\|\beta_{n}^{*}-\beta\right\|_{C\left([0, T] ; \mathbb{R}^{N}\right)}\left(\left\|y^{*}\right\|_{\tilde{X}_{[0, T]}}+\left\|y^{*}\right\|_{L^{1}\left(0, T ; L^{2}\right)}\right) \rightarrow 0 .
$$

Proceeding as in [1], (4.12), we have

$$
\begin{aligned}
& \left\|\left|y_{n}^{*}\right|^{\alpha-1} y_{n}^{*}-\left|y^{*}\right|^{\alpha-1} y^{*}\right\|_{L^{q^{\prime}}\left(0, t ; L^{p^{\prime}}\right)} \\
& \quad \leq \alpha t^{\theta}\left(\left\|y_{n}^{*}\right\|_{L^{q}\left(0, T ; L^{p}\right)}^{\alpha-1}+\left\|y^{*}\right\|_{L^{q}\left(0, T ; L^{p}\right)}^{\alpha-1}\right)\left\|y_{n}^{*}-y^{*}\right\|_{L^{q}\left(0, t ; L^{p}\right)} .
\end{aligned}
$$

Moreover, for $t \leq 1$,

$$
\begin{aligned}
& \left\|f\left(u_{n}^{*}\right) y_{n}^{*}-f\left(u^{*}\right) y^{*}\right\|_{L^{1}\left(0, t ; L^{2}\right)} \\
& \quad \leq t^{\frac{1}{2}} D\left(\left\|u_{n}^{*}-u^{*}\right\|_{L^{2}\left(0, T ; \mathbb{R}^{m}\right)}+\left\|y_{n}^{*}-y^{*}\right\|_{L^{\infty}\left(0, t ; L^{2}\right)}\right),
\end{aligned}
$$

where $D=2\left(\left|V_{0}\right|_{L^{\infty}}+\|V\|_{L^{\infty}\left(\mathbb{R}^{d} ; \mathbb{R}^{m}\right)}\left(|x|_{2}+D_{U}\right)\right)$. 
Hence,

$$
\begin{aligned}
\| y_{n}^{*}- & y^{*}\left\|_{L^{\infty}\left(0, t ; L^{2}\right)}+\right\| y_{n}^{*}-y^{*} \|_{L^{q}\left(0, t ; L^{p}\right)} \\
\leq & C_{T}\left[\alpha t^{\theta}\left(\left\|y_{n}^{*}\right\|_{L^{q}\left(0, T ; L^{p}\right)}^{\alpha-1}+\left\|y^{*}\right\|_{L^{q}\left(0, T ; L^{p}\right)}^{\alpha-1}\right)\left\|y_{n}^{*}-y^{*}\right\|_{L^{q}\left(0, t ; L^{p}\right)}\right. \\
& \left.\quad+t^{\frac{1}{2}} D\left\|u_{n}^{*}-u^{*}\right\|_{L^{2}\left(0, T ; \mathbb{R}^{m}\right)}+t^{\frac{1}{2}} D\left\|y_{n}^{*}-y^{*}\right\|_{L^{\infty}\left(0, t ; L^{2}\right)}\right]+w_{n}\left(y^{*}\right) \\
\leq & \left(t^{\theta}+t^{\frac{1}{2}}\right) C_{T} \widetilde{D}(T)\left(\left\|y_{n}^{*}-y^{*}\right\|_{L^{\infty}\left(0, t ; L^{2}\right)}+\left\|y_{n}^{*}-y^{*}\right\|_{L^{q}\left(0, t ; L^{p}\right)}\right. \\
& \left.+\left\|u_{n}^{*}-u^{*}\right\|_{L^{2}\left(0, T ; \mathbb{R}^{m}\right)}\right)+w_{n}\left(y^{*}\right)
\end{aligned}
$$

where $\widetilde{D}(T):=\alpha\left(\sup _{n \geq 1}\left\|y_{n}^{*}\right\|_{L^{q}\left(0, T ; L^{p}\right)}^{\alpha-1}+\left\|y^{*}\right\|_{L^{q}\left(0, T ; L^{p}\right)}^{\alpha-1}\right)+D<\infty, \mathbb{P}^{*}$-a.s. Choosing $t$ small enough and independent of $n$, such that $t^{\theta}+t^{\frac{1}{2}} \leq\left(2 \widetilde{D}(T) C_{T}\right)^{-1}$, we get that $\mathbb{P}^{*}$-a.s. as $n \rightarrow \infty$,

$$
\begin{aligned}
& \left\|y_{n}^{*}-y^{*}\right\|_{L^{\infty}\left(0, t ; L^{2}\right)}+\left\|y_{n}^{*}-y^{*}\right\|_{L^{q}\left(0, t ; L^{p}\right)} \\
& \leq\left\|u_{n}^{*}-u^{*}\right\|_{L^{2}\left(0, T ; \mathbb{R}^{m}\right)}+2 w_{n}\left(y^{*}\right) \rightarrow 0 .
\end{aligned}
$$

Since $t$ is independent of the initial data, iterating this procedure finite times we obtain (3.15), thereby completing the proof.

LEMmA 3.3. Let $X_{n}^{*},\left(\mathbb{X}_{T}^{*}\right)_{n}, \mathbb{X}_{1, n}^{*}, X^{*}, \mathbb{X}_{T}^{*}$ and $\mathbb{X}_{1}^{*}$ be as above, $n \geq 1$. We have, as $n \rightarrow \infty$,

$$
\mathbb{E}^{*} \operatorname{Re}\left(X_{n}^{*}(T),\left(\mathbb{X}_{T}^{*}\right)_{n}\right\rangle_{2} \rightarrow \mathbb{E}^{*} \operatorname{Re}\left\langle X^{*}(T), \mathbb{X}_{T}^{*}\right\rangle_{2}
$$

and

$$
\mathbb{E}^{*} \int_{0}^{T} \operatorname{Re}\left\langle X_{n}^{*}(t), \mathbb{X}_{1, n}^{*}(t)\right\rangle_{2} d t \rightarrow \mathbb{E}^{*} \int_{0}^{T} \operatorname{Re}\left\langle X^{*}(t), \mathbb{X}_{1}^{*}(t)\right\rangle_{2} d t
$$

Proof. By (3.11) and (3.8), $\mathbb{P}^{*}$-a.s., as $n \rightarrow \infty$,

$$
\begin{aligned}
\operatorname{Re}\left(X_{n}^{*}(T),\left(\mathbb{X}_{T}^{*}\right)_{n}\right\rangle_{2} & \rightarrow \operatorname{Re}\left\langle X^{*}(T), \mathbb{X}_{T}^{*}\right\rangle_{2}, \\
\int_{0}^{T} \operatorname{Re}\left\langle X_{n}^{*}(t), \mathbb{X}_{1, n}^{*}(t)\right\rangle_{2} d t & \rightarrow \int_{0}^{T} \operatorname{Re}\left\langle X^{*}(t), \mathbb{X}_{1}^{*}(t)\right\rangle_{2} d t
\end{aligned}
$$

Then, for any $\varepsilon \in(0,1)$ fixed, by the Young inequality $a b \leq \frac{1-\varepsilon}{2} a^{\frac{2}{1-\varepsilon}}+$ $\frac{1+\varepsilon}{2} b^{\frac{2}{1+\varepsilon}}$, we get

$$
\begin{aligned}
& \sup _{n \geq 1} \mathbb{E}^{*}\left|\left\langle X_{n}^{*}(T),\left(\mathbb{X}_{T}^{*}\right)_{n}\right\rangle_{2}\right|^{1+\varepsilon} \\
& \quad \leq \sup _{n \geq 1} \mathbb{E}^{*}\left|X_{n}^{*}(T)\right|_{2}^{1+\varepsilon}\left|\left(\mathbb{X}_{T}^{*}\right)_{n}\right|_{2}^{1+\varepsilon}
\end{aligned}
$$




$$
\begin{aligned}
& \leq \frac{1-\varepsilon}{2} \sup _{n \geq 1} \mathbb{E}^{*}\left|X_{n}^{*}(T)\right|_{2}^{\frac{2(1+\varepsilon)}{1-\varepsilon}}+\frac{1+\varepsilon}{2} \sup _{n \geq 1} \mathbb{E}^{*}\left|\left(\mathbb{X}_{T}^{*}\right)_{n}\right|_{2}^{2} \\
& =\frac{1-\varepsilon}{2}|x|_{2}^{\frac{2(1+\varepsilon)}{1-\varepsilon}}+\frac{1+\varepsilon}{2} \mathbb{E}\left|\mathbb{X}_{T}\right|_{2}^{2},
\end{aligned}
$$

which implies the uniform integrability of $\left\{\operatorname{Re}\left\langle X_{n}^{*}(T),\left(\mathbb{X}_{T}^{*}\right)_{n}\right\rangle_{2}\right\}_{n \geq 1}$, thereby yielding (3.22) by (3.24).

Similarly, for $\varepsilon \in(0,1)$ fixed, we have

$$
\begin{aligned}
\sup _{n \geq 1} & \mathbb{E}^{*}\left|\int_{0}^{T} \operatorname{Re}\left\langle X_{n}^{*}(t), \mathbb{X}_{1, n}^{*}(t)\right\rangle_{2} d t\right|^{1+\varepsilon} \\
& \leq \frac{1-\varepsilon}{2} \sup _{n \geq 1} \mathbb{E}^{*}\left\|X_{n}^{*}\right\|_{L^{2}\left(0, T ; L^{2}\right)}^{\frac{2(1+\varepsilon)}{1-\varepsilon}}+\frac{1+\varepsilon}{2} \sup _{n \geq 1} \mathbb{E}^{*}\left\|\mathbb{X}_{1, n}^{*}\right\|_{L^{2}\left(0, T ; L^{2}\right)}^{2} \\
& =\frac{1-\varepsilon}{2} T^{\frac{1+\varepsilon}{1-\varepsilon}}|x|_{2}^{\frac{2(1+\varepsilon)}{1-\varepsilon}}+\frac{1+\varepsilon}{2} \mathbb{E} \int_{0}^{T}\left|\mathbb{X}_{1}(t)\right|_{2}^{2} d t<\infty,
\end{aligned}
$$

which in view of (3.25) implies (3.23), as claimed.

Proof of TheOrem 2.5. By (3.12), we have

$$
\begin{aligned}
\Phi^{*}\left(u_{n}^{*}\right)= & \left(1+\gamma_{1}\right)|x|_{2}^{2}+\mathbb{E}^{*}\left|\left(\mathbb{X}_{T}^{*}\right)_{n}\right|_{2}^{2}+\gamma_{1} \mathbb{E}^{*} \int_{0}^{T}\left|\mathbb{X}_{1, n}^{*}(t)\right|_{2}^{2} d t \\
& -2 \mathbb{E}^{*} \operatorname{Re}\left(X_{n}^{*}(T),\left(\mathbb{X}_{T}^{*}\right)_{n}\right\rangle_{2}-2 \gamma_{1} \mathbb{E}^{*} \int_{0}^{T} \operatorname{Re}\left\langle X_{n}^{*}(t),\left.\mathbb{X}_{1, n}^{*}(t)\right|_{2} d t\right. \\
& +\gamma_{2} \mathbb{E}^{*} \int_{0}^{T}\left|u_{n}^{*}(t)\right|_{m}^{2} d t+\gamma_{3} \mathbb{E}^{*} \int_{0}^{T}\left|\left(u_{n}^{*}\right)^{\prime}(t)\right|_{m}^{2} d t .
\end{aligned}
$$

Note that, since the distributions of $\left(\mathbb{X}_{T}^{*}, \mathbb{X}_{1}^{*}\right)$ and $\left(\left(\mathbb{X}_{T}^{*}\right)_{n}, \mathbb{X}_{1, n}^{*}\right)$ coincide for $n \geq 1$, we have

$$
\begin{aligned}
& \mathbb{E}^{*}\left|\mathbb{X}_{T}^{*}\right|_{2}^{2}+\gamma_{1} \mathbb{E}^{*} \int_{0}^{T}\left|\mathbb{X}_{1}^{*}(t)\right|_{2}^{2} d t \\
& \quad=\lim _{n \rightarrow \infty}\left(\mathbb{E}^{*}\left|\left(\mathbb{X}_{T}^{*}\right)_{n}\right|_{2}^{2}+\gamma_{1} \mathbb{E}^{*} \int_{0}^{T}\left|\mathbb{X}_{1, n}^{*}(t)\right|_{2}^{2} d t\right) .
\end{aligned}
$$

Moreover, by (3.8) and the bounded dominated convergence theorem, it follows that

$$
\gamma_{2} \mathbb{E}^{*} \int_{0}^{T}\left|u_{n}^{*}(t)\right|_{m}^{2} d t \rightarrow \gamma_{2} \mathbb{E}^{*} \int_{0}^{T}\left|u^{*}(t)\right|_{m}^{2} d t \quad \text { as } n \rightarrow \infty,
$$

and by (3.9) and (3.10),

$$
\mathbb{E}^{*} \int_{0}^{T}\left|\left(u^{*}\right)^{\prime}(t)\right|_{m}^{2} d t \leq \liminf _{n \rightarrow \infty} \mathbb{E}^{*} \int_{0}^{T}\left|\left(u_{n}^{*}\right)^{\prime}(t)\right|_{m}^{2} d t
$$


Thus, taking into account Lemma 3.3, we obtain

$$
\Phi^{*}\left(u^{*}\right) \leq \liminf _{n \rightarrow \infty} \Phi^{*}\left(u_{n}^{*}\right)=\liminf _{n \rightarrow \infty} \Phi\left(u_{n}\right)=I,
$$

which completes the proof.

REMARK 3.4. The proofs above also show that, in the case $\gamma_{3}=0$, the objective functional $\Phi$ depends continuously on controls.

4. The directional derivative of function $\Phi$. This section is devoted to the calculation of the directional derivative of the function $\Phi$ on the convex set $\mathcal{U}_{\mathrm{ad}}$. Namely, one has

Proposition 4.1. Assume that $\gamma_{3}=0$ and that the conditions of Theorem 2.6 to hold. Then, for each $x \in L^{2}$ and all $u, v \in \mathcal{U}_{\mathrm{ad}}$, we have

$$
\lim _{\varepsilon \rightarrow 0} \frac{1}{\varepsilon}(\Phi(u+\varepsilon \tilde{u})-\Phi(u))=\mathbb{E} \int_{0}^{T} \eta(u)(t) \cdot \widetilde{u}(t) d t,
$$

where $\tilde{u}=v-u$, and

$$
\eta(u)=2\left(\gamma_{2} u-\operatorname{Im} \int_{\mathbb{R}^{d}} V(\xi) X^{u}(\xi) \overline{Y^{u}}(\xi) d \xi\right) .
$$

Here, $\left(Y^{u}, Z^{u}\right)$ is the solution to the dual backward stochastic equation (2.9).

To prove Proposition 4.1, we first study the equation of variation associated with Problem $(\mathrm{P})$, namely,

$$
\begin{aligned}
i d \varphi= & \Delta \varphi d t+\lambda h_{1}\left(X^{u}\right) \varphi d t+\lambda h_{2}\left(X^{u}\right) \bar{\varphi} d t-i \mu \varphi d t \\
& +V_{0} \varphi d t+u \cdot V \varphi d t+\tilde{u} \cdot V X^{u} d t+i \varphi d W(t), \\
\varphi(0)= & 0,
\end{aligned}
$$

where $\tilde{u}=v-u, u, v \in \mathcal{U}_{\mathrm{ad}}, X^{u}$ is the solution to (1.1), and $h_{j}\left(X^{u}\right), j=1,2$, are defined as in (2.10). The strong $H^{1}$-(and $L^{2}$-)solution to (4.3) can be defined similarly as in Definition 2.1 .

LEMMA 4.2. (i) Under Hypothesis (H0), for $u, v \in \mathcal{U}_{\mathrm{ad}}, \tilde{u}:=v-u$, there exists a unique strong $L^{2}$-solution $\varphi^{u, \widetilde{u}}$ to $(4.3)$ on $[0, T]$.

(ii) Under Hypothesis (H2), for any Strichartz pair $(p, q)$,

$$
\sup _{u, v \in \mathcal{U}_{\mathrm{ad}}}\left(\left\|\varphi^{u, \widetilde{u}}\right\|_{L^{\infty}\left(\Omega ; C\left([0, T] ; L^{2}\right)\right)}+\left\|\varphi^{u, \widetilde{u}}\right\|_{L^{\infty}\left(\Omega ; L^{q}\left(0, T ; L^{p}\right)\right)}\right)<\infty .
$$

Moreover, set $u_{\varepsilon}:=u+\varepsilon \tilde{u}$ and let $X^{u}$ and $X^{u_{\varepsilon}}$ be the corresponding solutions to (1.1) with the initial datum $x \in H^{1}$. Then

$$
\lim _{\varepsilon \rightarrow 0} \mathbb{E} \sup _{t \in[0, T]}\left|\varepsilon^{-1}\left(X^{u_{\varepsilon}}(t)-X^{u}(t)\right)-\varphi^{u, \tilde{u}}(t)\right|_{2}^{2}=0 .
$$


REMARK 4.3. In comparison with (ii), the weaker Hypothesis (H0) is sufficient for the pathwise existence and uniqueness of the solution to (4.3), thanks to the linear structure of (4.3). However, as mentioned in Section 2, Hypothesis (H2) is needed in order that the estimate (4.4) holds. The arguments presented below, particularly in the proof of estimate (4.4), will also be used in the analysis of the dual backward stochastic equation in the proof of Proposition 4.4 below.

Proof OF Lemma 4.2. (i) We set $z^{u, \widetilde{u}}:=e^{-W} \varphi^{u, \widetilde{u}}, \tilde{u}:=v-u$, and for simplicity, we omit the dependence of $u, \widetilde{u}$ in $z^{u, \tilde{u}}$ below. It follows from (4.3) that

$$
\begin{aligned}
d z= & A(t) z d t-\lambda i h\left(X^{u}, z\right) d t+f(u) z d t \\
& -i \tilde{u} \cdot V e^{-W} X^{u} d t, \quad t \in(0, T), \\
z(0)= & 0,
\end{aligned}
$$

where $A(t)$ is similar to $A_{n}^{*}(t)$ in (3.14), that is, $A(t)=-i(\Delta+b(t) \cdot \nabla+$ $c(t)), b(t)=2 \nabla W(t), c(t)=\sum_{j=1}^{d}\left(\partial_{j} W(t)\right)^{2}+\Delta W(t), h\left(X^{u}, z\right):=h_{1}\left(X^{u}\right) z+$ $h_{2}\left(X^{u}\right) e^{-2 i \operatorname{Im} W_{\bar{z}}}$ and $f(u):=-i\left(V_{0}+u \cdot V\right)$.

It is equivalent to prove the existence and uniqueness of the solution to (4.6) (see the proof of [1], Lemma 6.1).

To this purpose, we reformulate (4.6) in the mild form as

$$
\begin{aligned}
z(t)= & \int_{0}^{t} U(t, s)\left[-\lambda i h\left(X^{u}, z\right)(s)+f(u(s)) z(s)\right. \\
& \left.-i \tilde{u}(s) \cdot V e^{-W(s)} X^{u}(s)\right] d s,
\end{aligned}
$$

where $0 \leq t \leq T$, and $U(t, s), 0 \leq s, t \leq T$, are the evolution operators corresponding to the operator $A(t)$ (see [1], Lemma 3.3). Choose the Strichartz pair $(p, q)=\left(\alpha+1, \frac{4(\alpha+1)}{d(\alpha-1)}\right)$. Define the operator $F$ on $C\left([0, T] ; L^{2}\right) \cap L^{q}\left(0, T ; L^{p}\right)$ by

$$
F(\phi)(t):=\int_{0}^{t} U(t, s)\left[-\lambda i h\left(X^{u}, \phi\right)(s)+f(u(s)) \phi(s)-i \tilde{u} \cdot V e^{-W(s)} X^{u}(s)\right] d s,
$$

where $0 \leq t \leq T, \phi \in C\left([0, T] ; L^{2}\right) \cap L^{q}\left(0, T ; L^{p}\right)$. Set $\mathcal{Z}_{M_{1}}^{\tau_{1}}:=\left\{\phi \in C\left(\left[0, \tau_{1}\right]\right.\right.$; $\left.\left.L^{2}\right) \cap L^{q}\left(0, \tau_{1} ; L^{p}\right):\|\phi\|_{C\left(\left[0, \tau_{1}\right] ; L^{2}\right)}+\|\phi\|_{L^{q}\left(0, \tau_{1} ; L^{p}\right)} \leq M_{1}\right\}$, where $\tau_{1}$ and $M_{1}$ are two random variables to be determined later.

Note that, by Hölder's inequality, for any $\phi_{j} \in C\left([0, T] ; L^{2}\right) \cap L^{q}\left(0, T ; L^{p}\right)$, $j=1,2$,

$$
\left\|h\left(X^{u}, \phi_{1}\right)-h\left(X^{u}, \phi_{2}\right)\right\|_{L^{q^{\prime}}\left(0, t ; L^{p^{\prime}}\right)} \leq \alpha t^{\theta}\left\|X^{u}\right\|_{L^{q}\left(0, t ; L^{p}\right)}^{\alpha-1}\left\|\phi_{1}-\phi_{2}\right\|_{L^{q}\left(0, t ; L^{p}\right)},
$$

where $\theta=1-d(\alpha-1) / 4 \in(0,1)$, and

$$
\left\|f(u)\left(\phi_{1}-\phi_{2}\right)\right\|_{L^{1}\left(0, t ; L^{2}\right)} \leq t\|f(u)\|_{L^{\infty}\left(0, t ; L^{\infty}\right)}\left\|\phi_{1}-\phi_{2}\right\|_{C\left([0, t] ; L^{2}\right)} .
$$


Then let $R_{1}(t)=\alpha t^{\theta}\left\|X^{u}\right\|_{L^{q}\left(0, t ; L^{p}\right)}^{\alpha-1}+t\|f(u)\|_{L^{\infty}\left(0, t ; L^{\infty}\right)}, t \in[0, T]$. By the Strichartz estimates and the above estimates, we get for any $t \in[0, T]$,

$$
\begin{aligned}
& \left\|F\left(\phi_{1}\right)-F\left(\phi_{2}\right)\right\|_{C\left([0, t] ; L^{2}\right) \cap L^{q}\left(0, t ; L^{p}\right)} \\
& \quad \leq C_{t} R_{1}(t)\left\|\phi_{1}-\phi_{2}\right\|_{C\left([0, t] ; L^{2}\right) \cap L^{q}\left(0, t ; L^{p}\right)} .
\end{aligned}
$$

Similarly, for $\phi \in C\left([0, T] ; L^{2}\right) \cap L^{q}\left(0, T ; L^{p}\right), t \in[0, T]$,

$$
\begin{aligned}
& \|F(\phi)\|_{C\left([0, t] ; L^{2}\right) \cap L^{q}\left(0, t ; L^{p}\right)} \\
& \quad \leq C_{t} R_{1}(t)\|\phi\|_{C\left([0, t] ; L^{2}\right) \cap L^{q}\left(0, t ; L^{p}\right)}+C_{t}\left\|\tilde{u} \cdot V X^{u}\right\|_{L^{1}\left(0, t ; L^{2}\right)} .
\end{aligned}
$$

Setting $\tau_{1}=\inf \left\{t \in[0, T]: C_{t} R_{1}(t) \geq \frac{1}{2}\right\} \wedge T, M_{1}=2 C_{\tau_{1}}\left\|\tilde{u} \cdot V X^{u}\right\|_{L^{1}\left(0, \tau_{1} ; L^{2}\right)}$, it follows by (4.8) that $F$ is a contraction map in $\mathcal{Z}_{M_{1}}^{\tau_{1}}$, implying that there exists $\tilde{z}_{1} \in \mathcal{Z}_{M_{1}}^{\tau_{1}}$ such that $F\left(\tilde{z}_{1}\right)=\widetilde{z}_{1}$. Setting $z_{1}(\cdot):=\widetilde{z}_{1}\left(\cdot \wedge \tau_{1}\right)$ and using similar arguments as in [1], we deduce that $z_{1}$ is $\left(\mathcal{F}_{t}\right)$-adapted, continuous in $L^{2}$, and solves (4.6) on $\left[0, \tau_{1}\right]$, and $\left\|z_{1}\right\|_{C\left(\left[0, \tau_{1}\right] ; L^{2}\right) \cap L^{q}\left(0, \tau_{1} ; L^{p}\right)} \leq M_{1}$. We also note that $\tau_{1} \geq \sigma_{*}$, where

$$
\sigma_{*}:=\inf \left\{t \in[0, T] ; Z(t) \geq \frac{1}{2}\right\} \wedge T
$$

with $Z(t):=t^{\theta} \alpha C_{T}\left\|X^{u}\right\|_{L^{q}\left(0, T ; L^{p}\right)}^{\alpha-1}+t C_{T}\|f(u)\|_{L^{\infty}\left(0, T ; L^{\infty}\right)}, t \in[0, T]$.

Suppose that at the $n$ th-step $(n \geq 1)$ we have an increasing sequence of stopping times $\left\{\tau_{j}\right\}_{j=0}^{n}$ and an $L^{2}$-valued continuous $\left(\mathcal{F}_{t}\right)$-adapted process $z_{n}$ such that $\tau_{0}=$ $0, \tau_{j}-\tau_{j-1} \geq \sigma_{*}, 1 \leq j \leq n, z_{n}$ solves (4.6) on $\left[0, \tau_{n}\right], z_{n}(\cdot)=z_{n}\left(\cdot \wedge \tau_{n}\right)$, and

$$
\left\|z_{n}\right\|_{C\left(\left[0, \tau_{n}\right] ; L^{2}\right) \cap L^{q}\left(0, \tau_{n} ; L^{p}\right)} \leq \sum_{j=1}^{n}\left(2 C_{\tau_{n}}\right)^{n+1-j}\left\|\tilde{u} \cdot V X^{u}\right\|_{L^{1}\left(\tau_{j-1}, \tau_{j} ; L^{2}\right)} .
$$

Set $\mathcal{Z}_{M_{n+1}}^{\sigma_{n}}=\left\{\phi \in C\left(\left[0, \sigma_{n}\right] ; L^{2}\right) \cap L^{q}\left(0, \sigma_{n} ; L^{p}\right):\|\phi\|_{C\left(\left[0, \sigma_{n}\right] ; L^{2}\right)}+\right.$ $\left.\|\phi\|_{L^{q}\left(0, \sigma_{n} ; L^{p}\right)} \leq M_{n+1}\right\}$, where $\sigma_{n}$ and $M_{n+1}$ are random variables to be determined later. Define the operator $F_{n}$ on $C\left([0, T] ; L^{2}\right) \cap L^{q}\left(0, T ; L^{p}\right)$ by

$$
\begin{aligned}
F_{n}(\phi)(t):= & U\left(\tau_{n}+t, \tau_{n}\right) z_{n}\left(\tau_{n}\right)+\int_{0}^{t} U\left(\tau_{n}+t, \tau_{n}+s\right)\left[-\lambda i h\left(X^{u}\left(\tau_{n}+s\right), \phi(s)\right)\right. \\
& \left.+f\left(u\left(\tau_{n}+s\right)\right) \phi(s)-i \widetilde{u}\left(\tau_{n}+s\right) \cdot V e^{-W\left(\tau_{n}+s\right)} X^{u}\left(\tau_{n}+s\right)\right] d s,
\end{aligned}
$$

where $0 \leq t \leq T, \phi \in C\left([0, T] ; L^{2}\right) \cap L^{q}\left(0, T ; L^{p}\right)$.

Similarly, for any $\phi_{j} \in \mathcal{Z}_{M_{n+1}}^{\sigma_{n}}, j=1,2$,

$$
\begin{aligned}
& \left\|F_{n}\left(\phi_{1}\right)-F_{n}\left(\phi_{2}\right)\right\|_{C\left(\left[0, \sigma_{n}\right] ; L^{2}\right) \cap L^{q}\left(0, \sigma_{n} ; L^{p}\right)} \\
& \quad \leq C_{\tau_{n}+\sigma_{n}} R_{n+1}\left(\sigma_{n}\right)\left\|\phi_{1}-\phi_{2}\right\|_{C\left(\left[0, \sigma_{n}\right] ; L^{2}\right) \cap L^{q}\left(0, \sigma_{n} ; L^{p}\right)},
\end{aligned}
$$


where $R_{n+1}(t)=\alpha t^{\theta}\left\|X^{u}\right\|_{L^{q}\left(\tau_{n}, \tau_{n}+t ; L^{p}\right)}^{\alpha-1}+t\|f(u)\|_{L^{\infty}\left(\tau_{n}, \tau_{n}+t ; L^{\infty}\right)}, t \in\left[0, T-\tau_{n}\right]$, while for $\phi \in \mathcal{Z}_{M_{n+1}}^{\sigma_{n}}$, we have

$$
\begin{aligned}
\| F_{n}(\phi) & \|_{C\left(\left[0, \sigma_{n}\right] ; L^{2}\right) \cap L^{q}\left(0, \sigma_{n} ; L^{p}\right)} \\
\leq & C_{\tau_{n}+\sigma_{n}}\left|z_{n}\left(\tau_{n}\right)\right|_{2}+C_{\tau_{n}+\sigma_{n}}\left\|\tilde{u} \cdot V X^{u}\right\|_{L^{1}\left(\tau_{n}, \tau_{n}+\sigma_{n} ; L^{2}\right)} \\
& +C_{\tau_{n}+\sigma_{n}} R_{n+1}\left(\sigma_{n}\right)\|\phi\|_{C\left(\left[0, \sigma_{n}\right] ; L^{2}\right) \cap L^{q}\left(0, \sigma_{n} ; L^{p}\right)} \\
\leq & \frac{1}{2} \sum_{j=1}^{n+1}\left(2 C_{\tau_{n}+\sigma_{n}}\right)^{n+2-j}\left\|\tilde{u} \cdot V X^{u}\right\|_{L^{1}\left(\tau_{j-1}, \tau_{j} ; L^{2}\right)} \\
& +C_{\tau_{n}+\sigma_{n}} R_{n+1}\left(\sigma_{n}\right)\|\phi\|_{C\left(\left[0, \sigma_{n}\right] ; L^{2}\right) \cap L^{q}\left(0, \sigma_{n} ; L^{p}\right)} .
\end{aligned}
$$

Then let $\sigma_{n}(t):=\inf \left\{t \in\left[0, T-\tau_{n}\right]: C_{\tau_{n}+t} R_{n+1}(t) \geq \frac{1}{2}\right\} \wedge\left(T-\tau_{n}\right), \tau_{n+1}:=$ $\tau_{n}+\sigma_{n}$, and $M_{n+1}:=\sum_{j=1}^{n+1}\left(2 C_{\tau_{n+1}}\right)^{n+2-j}\left\|\tilde{u} \cdot V X^{u}\right\|_{L^{1}\left(\tau_{j-1}, \tau_{j} ; L^{2}\right)}$. It follows that $\tau_{n+1}-\tau_{n}=\sigma_{n} \geq \sigma_{*}, F_{n}$ is a contraction map in $\mathcal{Z}_{M_{n+1}}^{\sigma_{n}}$, and so there exists $\widetilde{z}_{n+1} \in$ $\mathcal{Z}_{M_{n+1}}^{\sigma_{n}}$ satisfying $F_{n}\left(\tilde{z}_{n+1}\right)=\widetilde{z}_{n+1}$. As in [1], letting

$$
z_{n+1}(t)= \begin{cases}z_{n}(t), & t \in\left[0, \tau_{n}\right] \\ \tilde{z}_{n+1}\left(\left(t-\tau_{n}\right) \wedge \sigma_{n}\right), & t \in\left(\tau_{n}, T\right]\end{cases}
$$

it follows that $z_{n+1}$ is continuous $\left(\mathcal{F}_{t}\right)$-adapted, satisfies $(4.6)$ on $\left[0, \tau_{n+1}\right], z_{n}(\cdot)=$ $z_{n}\left(\cdot \wedge \tau_{n+1}\right)$, and

$$
\left\|z_{n}\right\|_{C\left(\left[0, \tau_{n+1}\right] ; L^{2}\right) \cap L^{q}\left(0, \tau_{n+1} ; L^{p}\right)} \leq \sum_{j=1}^{n+1}\left(2 C_{\tau_{n+1}}\right)^{n+2-j}\left\|\tilde{u} \cdot V X^{u}\right\|_{L^{1}\left(\tau_{j-1}, \tau_{j} ; L^{2}\right)} .
$$

Iterating this procedure, since $\sigma_{n} \geq \sigma_{*}$, we see that after at most $\left[T / \sigma_{*}\right]+1$ steps the stopping time $\tau_{n}$ reaches $T$. Hence, $\mathbb{P}$-a.s. there exists a global solution (denoted by $z$ ) on $[0, T]$ which satisfies

$$
\|z\|_{C\left([0, T] ; L^{2}\right) \cap L^{q}\left(0, T ; L^{p}\right)}
$$

$$
\leq \sum_{j=1}^{\left[T / \sigma_{*}\right]+1}\left(2 C_{T}\right)^{\left[T / \sigma_{*}\right]+2-j}\left\|\tilde{u} \cdot V X^{u}\right\|_{L^{1}\left(\tau_{j-1}, \tau_{j} ; L^{2}\right)}
$$

As regards the uniqueness, given any two solutions $\varphi_{j}$, we set $z_{j}=e^{-W} \varphi_{j}$, $j=1,2$. Then, similar to (4.8), for any $s, t \in(0, T), s+t \leq T$, we have

$$
\begin{aligned}
\| z_{1}- & z_{2} \|_{C\left([s, s+t] ; L^{2}\right) \cap L^{q}\left(s, s+t ; L^{p}\right)} \\
\leq & C_{T}\left(\alpha t^{\theta}\left\|X^{u}\right\|_{L^{q}\left(0, T ; L^{p}\right)}^{\alpha-1}+t\|f(u)\|_{L^{\infty}\left(0, T ; L^{\infty}\right)}\right) \\
& \times\left\|z_{1}-z_{2}\right\|_{C\left([s, s+t] ; L^{2}\right) \cap L^{q}\left(s, s+t ; L^{p}\right)},
\end{aligned}
$$


which implies that $z_{1}=z_{2}$ on $[s, s+t], \mathbb{P}$-a.s., for $t$ sufficiently small and independent of $s$, thereby yielding the uniqueness by the arbitrariness of $s$.

(ii) Under Hypothesis (H2), the Strichartz coefficient $C_{T}$ is now a deterministic constant. Moreover, (2.5) and (4.9) imply that $\sigma_{*}$ has a deterministic lower bound, namely,

$$
\sigma_{*} \geq t_{*}:=\inf \left\{t \in[0, T]: Z^{*}(t) \geq T\right\} \wedge T, \quad \mathbb{P} \text {-a.s. }
$$

where

$$
Z^{*}(t):=\alpha C\left(t^{\theta}+t\right) \sup _{u \in \mathcal{U}_{\mathrm{ad}}}\left(\left\|X^{u}\right\|_{L^{\infty}\left(\Omega ; L^{q}\left(0, T ; L^{p}\right)\right)}^{\alpha-1}+\|f(u)\|_{L^{\infty}\left(\Omega ; L^{\infty}\left(0, T ; L^{\infty}\right)\right)}\right) .
$$

Thus, taking into account (4.10) and the uniform boundedness of $u, v \in \mathcal{U}_{\mathrm{ad}}$, we obtain (4.4).

Now, set $\widetilde{X}_{\varepsilon}^{u}:=\varepsilon^{-1}\left(X^{u_{\varepsilon}}-X^{u}\right)-\varphi$ and $\tilde{y}_{\varepsilon}^{u}:=e^{-W} \tilde{X}_{\varepsilon}^{u}$. We need to prove that

$$
\lim _{\varepsilon \rightarrow 0} \mathbb{E}\left\|\widetilde{y}_{\varepsilon}^{u}\right\|_{C\left([0, T] ; L^{2}\right)}^{2}=0 \text {. }
$$

To this purpose, note that

$$
\varepsilon^{-1}\left(u_{\varepsilon} \cdot V X^{u_{\varepsilon}}-u \cdot V X^{u}\right)=\tilde{u} \cdot V X^{u}+u_{\varepsilon} \cdot V\left(\tilde{X}_{\varepsilon}^{u}+\varphi\right),
$$

and

$$
\begin{aligned}
\varepsilon^{-1}\left(\left|X^{u_{\varepsilon}}\right|^{\alpha-1} X^{u_{\varepsilon}}-\left|X^{u}\right|^{\alpha-1} X^{u}\right)-\left(h_{1}\left(X^{u}\right) \varphi+h_{2}\left(X^{u}\right) \bar{\varphi}\right) \\
=\left(\int_{0}^{1} h_{1}\left(X_{u, r, \varepsilon}\right) d r\right) \tilde{X}_{\varepsilon}^{u}+\left(\int_{0}^{1} h_{2}\left(X_{u, r, \varepsilon}\right) d r\right) \overline{X_{\varepsilon}^{u}} \\
\quad+\varphi \int_{0}^{1}\left(h_{1}\left(X_{u, r, \varepsilon}\right)-h_{1}\left(X^{u}\right)\right) d r+\bar{\varphi} \int_{0}^{1}\left(h_{2}\left(X_{u, r, \varepsilon}\right)-h_{2}\left(X^{u}\right)\right) d r,
\end{aligned}
$$

where $X_{u, r, \varepsilon}=X^{u}+r\left(X^{u_{\varepsilon}}-X^{u}\right), r \in[0,1]$. For simplicity, set $R_{j}(\varepsilon):=$ $\int_{0}^{1}\left(h_{j}\left(X_{u, r, \varepsilon}\right)-h_{j}\left(X^{u}\right)\right) d r, j=1,2$, and $R(\varepsilon, \varphi):=-i\left(\lambda R_{1}(\varepsilon) \varphi+\lambda R_{2}(\varepsilon) \bar{\varphi}+\right.$ $\varepsilon \widetilde{u} \cdot V \varphi)$.

Then, by (1.1) and (4.3), $\widetilde{X}_{\varepsilon}^{u}$ satisfies the equation:

$$
\begin{aligned}
d \widetilde{X}_{\varepsilon}^{u}= & -i \Delta \widetilde{X}_{\varepsilon}^{u} d t-\lambda i \int_{0}^{1} h_{1}\left(X_{u, r, \varepsilon}\right) d r \widetilde{X}_{\varepsilon}^{u} d t \\
& -\lambda i \int_{0}^{1} h_{2}\left(X_{u, r, \varepsilon}\right) d r \overline{\widetilde{X}_{\varepsilon}^{u}} d t \\
& -\left(\mu+i V_{0}+i u_{\varepsilon} \cdot V\right) \tilde{X}_{\varepsilon}^{u} d t+R(\varepsilon, \varphi) d t+\widetilde{X}_{\varepsilon}^{u} d W(t) .
\end{aligned}
$$

This yields

$$
\begin{aligned}
d \widetilde{y}_{\varepsilon}^{u}= & -i \Delta \tilde{y}_{\varepsilon}^{u} d t-\lambda i \int_{0}^{1} h\left(X_{u, r, \varepsilon}, \tilde{y}_{\varepsilon}^{u}\right) d r d t \\
& +f\left(u_{\varepsilon}\right) \tilde{y}_{\varepsilon}^{u} d t+e^{-W} R(\varepsilon, \varphi) d t
\end{aligned}
$$


where $h\left(X_{u, r, \varepsilon}, \tilde{y}_{\varepsilon}^{u}\right)$ and $f\left(u_{\varepsilon}\right)$ are similar as in (4.6), with $X^{u}, z, u$ replaced by $X_{u, r, \varepsilon}, \tilde{y}_{\varepsilon}^{u}$ and $u_{\varepsilon}$, respectively.

Choose the Strichartz pair $(p, q)=\left(\alpha+1, \frac{4(\alpha+1)}{d(\alpha-1)}\right)$. Then, by Strichartz's estimates, Hölder's inequality and Minkowski's inequality, we have

$$
\begin{aligned}
& \left\|\tilde{y}_{\varepsilon}^{u}\right\|_{C\left([0, t] ; L^{2}\right)}+\left\|\tilde{y}_{\varepsilon}^{u}\right\|_{L^{q}\left(0, t ; L^{p}\right)} \\
& \leq C \int_{0}^{1}\left\|h\left(X_{u, r, \varepsilon}, \tilde{y}_{\varepsilon}^{u}\right)\right\|_{L^{q^{\prime}\left(0, t, L^{p^{\prime}}\right)}} d r+C t\left\|f\left(u_{\varepsilon}\right)\right\|_{L^{\infty}\left(0, T ; L^{\infty}\right)}\left\|\widetilde{y}_{\varepsilon}^{u}\right\|_{C\left([0, t] ; L^{2}\right)} \\
& \quad+C\|R(\varepsilon, \varphi)\|_{L^{1}\left(0, t ; L^{2}\right)+L^{q^{\prime}}\left(0, t ; L^{p^{\prime}}\right)} .
\end{aligned}
$$

Note that

$$
\begin{aligned}
& \left\|h\left(X_{u, r, \varepsilon}, \widetilde{y}_{\varepsilon}^{u}\right)\right\|_{L^{q^{\prime}}\left(0, t, L^{p^{\prime}}\right)} \\
& \quad \leq \alpha t^{\theta}\left\|\widetilde{y}_{\varepsilon}^{u}\right\|_{L^{q}\left(0, t ; L^{p}\right)}\left\|X_{u, r, \varepsilon}\right\|_{L^{q}\left(0, t ; L^{p}\right)}^{\alpha-1} \\
& \quad \leq \alpha\left(1 \vee 2^{\alpha-1}\right) t^{\theta}\left\|\widetilde{y}_{\varepsilon}^{u}\right\|_{L^{q}\left(0, t ; L^{p}\right)}\left(\left\|X^{u_{\varepsilon}}\right\|_{L^{q}\left(0, T ; L^{p}\right)}^{\alpha-1}+\left\|X^{u}\right\|_{L^{q}\left(0, T ; L^{p}\right)}^{\alpha-1}\right),
\end{aligned}
$$

where $\theta=1-d(\alpha-1) / 4>0$. Then

$$
\begin{aligned}
& \left\|\tilde{y}_{\varepsilon}^{u}\right\|_{C\left([0, t] ; L^{2}\right)}+\left\|\tilde{y}_{\varepsilon}^{u}\right\|_{L^{q}\left(0, t ; L^{p}\right)} \\
& \leq C D_{3}(T)\left(t^{\theta}+t\right)\left(\left\|\tilde{y}_{\varepsilon}^{u}\right\|_{C\left([0, t] ; L^{2}\right)}+\left\|\tilde{y}_{\varepsilon}^{u}\right\|_{L^{q}\left(0, t ; L^{p}\right)}\right) \\
& \quad+C\|R(\varepsilon, \varphi)\|_{L^{1}\left(0, T ; L^{2}\right)+L^{q^{\prime}}\left(0, T ; L^{p^{\prime}}\right)},
\end{aligned}
$$

where

$$
D_{3}(T)=\alpha 2^{\alpha+1} \sup _{\varepsilon \in[0,1]}\left(\left\|X^{u_{\varepsilon}}\right\|_{L^{\infty}\left(\Omega ; L^{q}\left(0, T ; L^{p}\right)\right)}^{\alpha-1}+\left\|f\left(u_{\varepsilon}\right)\right\|_{L^{\infty}\left(\Omega ; L^{\infty}\left(0, T ; L^{\infty}\right)\right)}\right) .
$$

Using similar iterating arguments as in the proof of (4.4), we obtain

$$
\sup _{u \in \mathcal{U}_{\mathrm{ad}}}\left(\left\|\widetilde{y}_{\varepsilon}^{u}\right\|_{C\left([0, T] ; L^{2}\right)}+\left\|\widetilde{y}_{\varepsilon}^{u}\right\|_{L^{q}\left(0, T ; L^{p}\right)}\right) \leq C(T)\|R(\varepsilon, \varphi)\|_{L^{1}\left(0, T ; L^{2}\right)+L^{q^{\prime}}\left(0, T ; L^{p^{\prime}}\right)}
$$

with $C(T) \in L^{\infty}(\Omega)$.

Thus, by Hölder's inequality,

$$
\begin{aligned}
\mathbb{E}\left\|\widetilde{y}_{\varepsilon}^{u}\right\|_{C\left([0, T] ; L^{2}\right)}^{2}+\mathbb{E}\left\|\widetilde{y}_{\varepsilon}^{u}\right\|_{L^{q}\left(0, T ; L^{p}\right)}^{2} \\
\leq C(T) \mathbb{E}\|R(\varepsilon, \varphi)\|_{L^{1}\left(0, T ; L^{2}\right)+L^{q^{\prime}}\left(0, T ; L^{p^{\prime}}\right)}^{2} \\
\leq C(T)\left(\varepsilon^{2} D_{U}^{2} T^{2}\|V\|_{L^{\infty}\left(0, T ; \mathbb{R}^{m}\right)}^{2} \mathbb{E}\|\varphi\|_{C\left([0, T] ; L^{2}\right)}^{2}\right. \\
\left.\quad+\sum_{j=1}^{2} \mathbb{E}\left\|R_{j}(\varepsilon) \varphi\right\|_{L^{q^{\prime}}\left(0, T ; L^{p^{\prime}}\right)}^{2}\right) .
\end{aligned}
$$


Therefore, in order to prove (4.11), we only need to show that

$$
\mathbb{E}\left\|R_{j}(\varepsilon) \varphi\right\|_{L^{q^{\prime}}\left(0, T ; L^{p^{\prime}}\right)}^{2} \rightarrow 0 \quad \text { as } \varepsilon \rightarrow 0, j=1,2 .
$$

Below, we prove (4.15) only for $R_{1}(\varepsilon)$, but the argument applies as well to $R_{2}(\varepsilon)$. As in the proof of (3.15), we get

$$
\left\|X^{u_{\varepsilon}}-X^{u}\right\|_{C\left([0, T] ; L^{2}\right)}+\left\|X^{u_{\varepsilon}}-X^{u}\right\|_{L^{q}\left(0, T ; L^{p}\right)} \rightarrow 0
$$

$$
\text { as } \varepsilon \rightarrow 0, \mathbb{P} \text {-a.s. }
$$

Note that

$$
\begin{aligned}
& h_{1}\left(X_{u, r, \varepsilon}\right)-h_{1}\left(X^{u}\right) \\
& =\int_{0}^{1} \partial_{z} h_{1}\left(X^{u}+r^{\prime}\left(X_{u, r, \varepsilon}-X^{u}\right)\right) d r^{\prime}\left(X_{u, r, \varepsilon}-X^{u}\right) \\
& \quad+\int_{0}^{1} \partial_{\bar{z}} h_{1}\left(X^{u}+r^{\prime}\left(X_{u, r, \varepsilon}-X^{u}\right)\right) d r^{\prime}\left(\overline{X_{u, r, \varepsilon}}-\overline{X^{u}}\right) .
\end{aligned}
$$

Since $\left|\partial_{z} h_{1}(z)\right|+\left|\partial_{z} h_{1}(z)\right| \leq C|z|^{\alpha-2}$ for $z \in \mathbb{C}$, using the Minkowski inequality and the Hölder inequality we get that $\mathbb{P}$-a.s. for each $r \in[0,1]$,

$$
\begin{aligned}
& \left\|h_{1}\left(X_{u, r, \varepsilon}\right)-h_{1}\left(X^{u}\right)\right\|_{L^{\frac{q}{\alpha-1}}\left(0, T ; L^{\frac{p}{\alpha-1}}\right)} \\
& \quad \leq C \int_{0}^{1}\left\|X^{u}+r^{\prime}\left(X_{u, r, \varepsilon}-X^{u}\right)\right\|_{L^{q}\left(0, T ; L^{p}\right)}^{\alpha-2} d r^{\prime}\left\|X_{u, r, \varepsilon}-X^{u}\right\|_{L^{q}\left(0, T ; L^{p}\right)} \\
& \quad \leq C \sup _{\varepsilon \in[0,1]}\left\|X^{u_{\varepsilon}}\right\|_{L^{q}\left(0, T ; L^{p}\right)}^{\alpha-2}\left\|X^{u_{\varepsilon}}-X^{u}\right\|_{L^{q}\left(0, T ; L^{p}\right)} \rightarrow 0 \quad \text { as } \varepsilon \rightarrow 0,
\end{aligned}
$$

where we also used $\alpha \geq 2$ and the last step is due to (4.16).

Thus, using the Hölder inequality combined with the Minkowski inequality and the bounded dominated convergence theorem we obtain that

$$
\begin{aligned}
& \left\|R_{1}(\varepsilon) \varphi\right\|_{L^{q^{\prime}}\left(0, T ; L^{p^{\prime}}\right)} \\
& \quad \leq T^{\theta}\|\varphi\|_{L^{q}\left(0, T ; L^{p}\right)} \int_{0}^{1}\left\|h_{1}\left(X_{u, r, \varepsilon}\right)-h_{1}\left(X^{u}\right)\right\|_{L^{\frac{q}{\alpha-1}\left(0, T ; L^{\alpha-1}\right)}} d r \rightarrow 0 \\
& \quad \text { as } \varepsilon \rightarrow 0, \mathbb{P} \text {-a.s. }
\end{aligned}
$$

Moreover, taking into account (4.18), (2.5) and (4.4), we have

$$
\left\|R_{1}(\varepsilon) \varphi\right\|_{L^{q^{\prime}}\left(0, T ; L^{p^{\prime}}\right)} \leq C T^{\theta} \sup _{\varepsilon \in[0,1]}\left\|X^{u_{\varepsilon}}\right\|_{L^{q}\left(0, T ; L^{p}\right)}^{\alpha-1}\|\varphi\|_{L^{q}\left(0, T ; L^{p}\right)} \in L^{\infty}(\Omega),
$$

which along with (4.19) and the bounded dominated convergence theorem yields (4.15) for $j=1$. Therefore, the proof is complete.

We shall prove now the following result. 
Proposition 4.4. (i) Assume Hypothesis (H2) and that $\mathbb{X}_{T} \in L^{2+v}\left(\Omega ; L^{2}\right)$, $\mathbb{X}_{1} \in L^{2+v}\left(\Omega ; L^{2}\left(0, T ; L^{2}\right)\right)$ for some small $v \in(0,1)$.

Then there exists a unique $\left(\mathcal{F}_{t}\right)$-adapted solution $\left(Y^{u}, Z^{u}\right)$ to (2.9) corresponding to $u \in \mathcal{U}_{\mathrm{ad}}$, satisfying for any Stirchartz pair $(p, q)$,

$$
\sup _{u \in \mathcal{U}_{\mathrm{ad}}}\left(\left\|Y^{u}\right\|_{L^{2+v}\left(\Omega ; C\left([0, T] ; L^{2}\right)\right)}+\left\|Y^{u}\right\|_{L^{2+v}\left(\Omega ; L^{q}\left(0, T ; L^{p}\right)\right)}\right)<\infty
$$

and

$$
\sup _{u \in \mathcal{U}_{\mathrm{ad}}}\left\|Z_{k}^{u}\right\|_{L^{2+v}\left(\Omega ; L^{2}\left(0, T ; L^{2}\right)\right)}<\infty, \quad 1 \leq k \leq N .
$$

(ii) Assume in addition that $\mathbb{X}_{T} \in L^{2+v}\left(\Omega ; H^{1}\right), \mathbb{X}_{1} \in L^{2+v}\left(\Omega ; L^{2}\left(0, T ; H^{1}\right)\right)$ for some small $v \in(0,1)$. Then, for any $\rho \in[2,2+v)$ and any Strichartz pair $(p, q)$, we have

$$
\sup _{u \in \mathcal{U}_{\mathrm{ad}}}\left(\left\|Y^{u}\right\|_{L^{\rho}\left(\Omega ; C\left([0, T] ; H^{1}\right)\right)}+\left\|Y^{u}\right\|_{L^{\rho}\left(\Omega ; L^{q}\left(0, T ; W^{1, p}\right)\right)}\right)<\infty
$$

and

$$
\sup _{u \in \mathcal{U}_{\mathrm{ad}}}\left\|Z_{k}^{u}\right\|_{L^{\rho}\left(\Omega ; L^{2}\left(0, T ; H^{1}\right)\right)}<\infty, \quad 1 \leq k \leq N
$$

As mentioned in Section 2, the main difficulty in the analysis of the backward stochastic equation comes from the singular term $\lambda i h_{2}\left(X^{u}\right) \bar{Y}$. The proof of Proposition 4.4 follows the following steps.

First, we will consider the truncated approximating equation (4.24) and introduce the dual equation (4.26) below, which are related by the formula (4.29). Then the uniform estimate (4.28) for the dual solutions imply, via duality arguments, those for the approximating solutions $\left\{Y_{n}\right\}$ [see (4.31) below], which in turn imply the uniform estimates for $\left\{Z_{n}\right\}$ [see (4.35) below]. Consequently, in view of the linear structure of (4.24), one can pass to the limit and obtain the well-posedness of problem (2.9) as well as the estimates (4.20) and (4.21).

Analogous arguments are applicable to prove estimates (4.22) and (4.23) in Sobolev spaces, which requires the condition $\alpha \geq 2$ and the integrability conditions on $\mathbb{X}_{T}$ and $\mathbb{X}_{1}$ in Sobolev spaces.

PROOF. (i). Let $g$ be a radial smooth cut-off function such that $g=1$ on $B_{1}(\mathbb{R})$, and $g=0$ on $B_{2}^{c}(\mathbb{R})$. For $j=1,2$, set $h_{j, n}\left(X^{u}\right):=g\left(\frac{\left|X^{u}\right|}{n}\right) h_{j}\left(X^{u}\right)$. Note that $\left|h_{1, n}\left(X^{u}\right)\right|+\left|h_{2, n}\left(X^{u}\right)\right| \leq \alpha 2^{\alpha-1}|g|_{L^{\infty}} n^{\alpha-1}$.

Consider the approximating backward stochastic equation:

$$
\begin{aligned}
d Y_{n}= & -i \Delta Y_{n} d t-\lambda i h_{1, n}\left(X^{u}\right) Y_{n} d t+\lambda i h_{2, n}\left(X^{u}\right) \overline{Y_{n}} d t \\
& +\mu Y_{n} d t-i V_{0} Y_{n} d t
\end{aligned}
$$




$$
\begin{aligned}
& -i u \cdot V Y_{n} d t+\gamma_{1}\left(X^{u}-\mathbb{X}_{1}\right) d t-\sum_{k=1}^{N} \overline{\mu_{k}} e_{k} Z_{k, n} d t \\
& +\sum_{k=1}^{N} Z_{k, n} d \beta_{k}(t), \\
Y_{n}(T)=- & \left(X^{u}(T)-\mathbb{X}_{T}\right) .
\end{aligned}
$$

By standard theory for stochastic backward infinite dimensional equations (see, e.g., $[14,17,28])$, it follows that there exists a unique $\left(\mathcal{F}_{t}\right)$-adapted solution $\left(Y_{n}, Z_{n}\right) \in L^{2}\left(\Omega ; C\left([0, T] ; L^{2}\right)\right) \times\left(L_{\text {ad }}^{2}\left(0, T ; L^{2}\left(\Omega ; L^{2}\right)\right)\right)^{N}$ to $(4.24)$.

In order to pass to the limit $n \rightarrow \infty$, we are going to obtain uniform estimates of $Y_{n}$ in the space $\mathcal{Y}_{\rho_{v}}:=L^{\rho_{\nu}}\left(\Omega ; L^{\infty}\left(0, T ; L^{2}\right)\right) \cap L^{\rho_{\nu}}\left(\Omega ; L^{q}\left(0, T ; L^{p}\right)\right)$ with $\rho_{v}:=$ $2+v$ and $(p, q)=\left(\alpha+1, \frac{4(\alpha+1)}{d(\alpha-1)}\right)$.

To this purpose, for each $n \geq 1$, define the functional $\Lambda_{n}$ on the space $L^{\infty}(\Omega \times$ $\left.(0, T) \times \mathbb{R}^{d}\right)$,

$$
\begin{aligned}
\Lambda_{n}(\Psi):= & \mathbb{E} \operatorname{Re}\left\langle X^{u}(T)-\mathbb{X}_{T}, \psi_{n}(T)\right\rangle_{2} \\
& +\gamma_{1} \mathbb{E} \int_{0}^{T} \operatorname{Re}\left\langle X^{u}(t)-\mathbb{X}_{1}(t), \psi_{n}(t)\right\rangle_{2} d t,
\end{aligned}
$$

where $\Psi \in L^{\infty}\left(\Omega \times(0, T) \times \mathbb{R}^{d}\right), X^{u}$ is the solution to (1.1), and $\psi_{n}$ satisfies

$$
\begin{aligned}
d \psi_{n}= & -i \Delta \psi_{n} d t-\lambda i h_{1, n}\left(X^{u}\right) \psi_{n} d t-\lambda i h_{2, n}\left(X^{u}\right) \overline{\psi_{n}} d t-\mu \psi_{n} d t \\
& -i V_{0} \psi_{n} d t-i u \cdot V \psi_{n} d t-\Psi d t+\psi_{n} d W(t) \\
\psi_{n}(0)= & 0
\end{aligned}
$$

[Note that, (4.26) is similar to (4.3) but with $\Psi$ in place of $i \widetilde{u} \cdot V X^{u}$.]

Since $\left|h_{j, n}\left(X^{u}\right)\right| \leq \alpha|g|_{L^{\infty}}\left|X^{u}\right|^{\alpha-1}, j=1,2$, arguing as in the proof of Lemma 4.2 we infer that there exists a unique strong $L^{2}$-solution $\psi_{n}$ to (4.26) on $[0, T]$, satisfying

$$
\begin{gathered}
\sup _{n}\left(\left\|\psi_{n}\right\|_{C\left([0, T] ; L^{2}\right)}+\left\|\psi_{n}\right\|_{L^{q}\left(0, T ; L^{p}\right)}\right) \\
\leq C(T)\|\Psi\|_{L^{1}\left(0, T ; L^{2}\right)+L^{q^{\prime}}\left(0, T ; L^{p^{\prime}}\right)},
\end{gathered}
$$

where $C(T) \in L^{\infty}(\Omega)$ is independent of $n$ and $\Psi$. It follows also that for $\rho>1$,

$$
\sup _{n \geq 1}\left\|\psi_{n}\right\|_{\mathcal{Y}_{\rho^{\prime}}} \leq C(\rho, T)\|\Psi\|_{\mathcal{Y}_{\rho^{\prime}}^{\prime}},
$$

where $C(\rho, T)$ is independent of $n$ and $\Psi$ and $\mathcal{Y}_{\rho^{\prime}}^{\prime}:=L^{\rho^{\prime}}\left(\Omega ; L^{1}\left(0, T ; L^{2}\right)\right)+$ $L^{\rho^{\prime}}\left(\Omega ; L^{q^{\prime}}\left(0, T ; L^{p^{\prime}}\right)\right)$. 
Moreover, by Itô's formula, for every $n \geq 1$ and $\Psi \in L^{\infty}\left(\Omega \times(0, T) \times \mathbb{R}^{d}\right)$, we have

$$
\Lambda_{n}(\Psi)=\mathbb{E} \int_{0}^{T} \operatorname{Re}\left\langle\Psi, Y_{n}\right\rangle_{2} d t
$$

Thus, since $\left|X^{u}(t)\right|_{2}$ is $\mathbb{P}$-a.s. conserved and by estimates (4.25), (4.28), we have

$$
\begin{aligned}
\left|\Lambda_{n}(\Psi)\right| \leq & \gamma_{1}\left\|X^{u}-\mathbb{X}_{1}\right\|_{L^{\rho_{\nu}}\left(\Omega ; L^{2}\left(0, T ; L^{2}\right)\right)}\left\|\psi_{n}\right\|_{L^{\rho_{\nu}^{\prime}\left(\Omega ; L^{2}\left(0, T ; L^{2}\right)\right)}} \\
& \quad\left\|X^{u}(T)-\mathbb{X}_{T}\right\|_{L^{\rho_{\nu}}\left(\Omega ; L^{2}\right)}\left\|\psi_{n}(T)\right\|_{L_{\nu}^{\rho_{\nu}^{\prime}\left(\Omega ; L^{2}\right)}} \\
\leq & C\|\Psi\|_{\mathcal{Y}_{\rho_{\nu}^{\prime}}^{\prime}},
\end{aligned}
$$

where $C$ is independent of $n$. Since $L^{\infty}\left(\Omega \times(0, T) \times \mathbb{R}^{d}\right)$ is dense in $\mathcal{Y}_{\rho_{v}^{\prime}}^{\prime}$ and $\mathcal{Y}_{\rho_{v}}$ is the dual space of $\mathcal{Y}_{\rho_{v}^{\prime}}^{\prime}$, it follows by (4.29), (4.30) that

$$
\sup _{n \geq 1}\left\|Y_{n}\right\|_{\mathcal{Y}_{\rho_{v}}}<\infty
$$

Hence, there exists $\tilde{Y} \in \mathcal{Y}_{\rho_{\nu}}$, such that along a subsequence of $\{n\} \rightarrow \infty$ (still denoted by $\{n\})$,

$$
Y_{n} \rightarrow \tilde{Y} \quad \text { weak }^{*} \text { in } \mathcal{Y}_{\rho_{\nu}} .
$$

Note that, for each $j=1,2, h_{j, n}\left(X^{u}\right) \rightarrow h_{j}\left(X^{u}\right), d \mathbb{P} \otimes d t \otimes d \xi$-a.e., and $\sup _{n \geq 1}\left|h_{j, n}\left(X^{u}\right)\right| \leq \alpha|g|_{L^{\infty}}\left|X^{u}\right|^{\alpha-1} \in L^{2 \rho_{v}^{\prime}}\left(\Omega ; L^{\frac{q}{\alpha-1}}\left(0, T ; L^{\frac{p}{\alpha-1}}\right)\right)$. Hence, by the dominated convergence theorem,

(4.33) $h_{j, n}\left(X^{u}\right) \rightarrow h_{j}\left(X^{u}\right) \quad$ in $L^{2 \rho_{\nu}^{\prime}}\left(\Omega ; L^{\frac{q}{\alpha-1}}\left(0, T ; L^{\frac{p}{\alpha-1}}\right)\right)$, as $n \rightarrow \infty$.

Since $\frac{1}{\left(2 \rho_{v}^{\prime}\right)^{\prime}}=\frac{1}{\rho_{v}}+\frac{1}{2 \rho_{v}^{\prime}}$, from Hölder's inequality, (4.32) and (4.33) it follows that

$$
h_{1, n}\left(X^{u}\right) Y_{n} \rightarrow h_{1}\left(X^{u}\right) \tilde{Y}, \quad h_{2, n}\left(X^{u}\right) \overline{Y_{n}} \rightarrow h_{2}\left(X^{u}\right) \overline{\widetilde{Y}},
$$

weakly in $L^{\left(2 \rho_{v}^{\prime}\right)^{\prime}}\left(\Omega ; L^{\frac{q}{\alpha}}\left(0, T ; L^{p^{\prime}}\right)\right)$.

Moreover, we claim that for $1 \leq k \leq N$,

$$
\sup _{n \geq 1}\left\|Z_{k, n}\right\|_{L^{\rho_{\nu}}\left(\Omega ; L^{2}\left(0, T ; L^{2}\right)\right)} \leq C<\infty .
$$

In particular, for each $1 \leq k \leq N$, there exists $Z_{k}^{u} \in L^{\rho_{\nu}}\left(\Omega ; L^{2}\left(0, T ; L^{2}\right)\right)$ such that (selecting a further subsequence if necessary)

$$
Z_{k, n} \rightarrow Z_{k}^{u} \quad \text { weakly in } L^{2}\left(\Omega ; L^{2}\left(0, T ; L^{2}\right)\right) .
$$

Since $v \mapsto \int^{T} v d \beta_{k}(s)$ is a bounded linear operator in $L^{2}\left(\Omega ; L^{2}\left(0, T ; L^{2}\right)\right)$, it follows that for each $1 \leq k \leq N$,

$$
\int^{T} Z_{k, n} d \beta_{k}(s) \rightarrow \int^{T} Z_{k}^{u} d \beta_{k}(s) \quad \text { weakly in } L^{2}\left(\Omega ; L^{2}\left(0, T ; L^{2}\right)\right)
$$


To prove (4.35), we apply Itô's formula to (4.24) to get that for $\eta>0$,

$$
\begin{aligned}
e^{\eta t}\left|Y_{n}(t)\right|_{2}^{2} & \\
= & e^{\eta T}\left|X^{u}(T)-\mathbb{X}_{T}\right|_{2}^{2}-\eta \int_{t}^{T} e^{\eta s}\left|Y_{n}\right|_{2}^{2} d s \\
& +2 \lambda \int_{t}^{T} \operatorname{Im} \int e^{\eta s} h_{2, n}\left(X^{u}\right) \overline{Y_{n}^{2}} d \xi d s-2 \int_{t}^{T} \int e^{\eta s} \mu\left|Y_{n}\right|^{2} d \xi d s \\
& -2 \gamma_{1} \int_{t}^{T} \operatorname{Re} \int e^{\eta s} Y_{n}\left(\overline{X^{u}}-\overline{\mathbb{X}_{1}}\right) d \xi d s \\
& +2 \sum_{k=1}^{N} \int_{t}^{T} \operatorname{Re} \int e^{\eta s} \mu_{k} e_{k} Y_{n} \overline{Z_{k, n}} d \xi d s \\
& -\sum_{k=1}^{N} \int_{t}^{T} e^{\eta s}\left|Z_{k, n}\right|_{2}^{2} d s-2 \sum_{k=1}^{N} \int_{t}^{T} \operatorname{Re} \int e^{\eta s} Y_{n} \overline{Z_{k, n}} d \xi d \beta_{k}(s) \\
= & e^{\eta T}\left|X^{u}(T)-\mathbb{X}_{T}\right|_{2}^{2}-\eta \int_{t}^{T} e^{\eta s}\left|Y_{n}\right|_{2}^{2} d s+\sum_{j=1}^{6} K_{j}(t) .
\end{aligned}
$$

Note that, since $\left|h_{2, n}\left(X^{u}\right)\right| \leq \alpha|g|_{L^{\infty}}\left|X^{u}\right|^{\alpha-1}$, by Hölder's inequality,

$$
\left|K_{1}(t)\right| \leq \alpha|g|_{L^{\infty}} e^{\eta T} T^{\theta}\left\|X^{u}\right\|_{L^{q}\left(0, T ; L^{p}\right)}^{\alpha-1}\left\|Y_{n}\right\|_{L^{q}\left(0, T ; L^{p}\right)}^{2},
$$

where $\theta=1-d(\alpha-1) / 4 \in(0,1)$.

Moreover, using $a b \leq c a^{2}+c^{-1} b^{2}, c>0$, we get

$$
\begin{aligned}
\sum_{j=2}^{4}\left|K_{j}(t)\right| \leq & \left(2|\mu|_{\infty}+2 \gamma_{1}+8 \sum_{k=1}^{N}\left|\mu_{k} e_{k}\right|_{L^{\infty}}^{2}\right) \int_{t}^{T} e^{\eta s}\left|Y_{n}\right|_{2}^{2} d s \\
& +2 \gamma_{1} \int_{t}^{T} e^{\eta s}\left|X^{u}-\mathbb{X}_{1}\right|_{2}^{2} d s+\frac{1}{2} \int_{t}^{T} e^{\eta s}\left|Z_{k, n}\right|_{2}^{2} d s
\end{aligned}
$$

Thus, choosing $\eta>2|\mu|_{\infty}+2 \gamma_{1}+8 \sum_{k=1}^{N}\left|\mu_{k} e_{k}\right|_{L^{\infty}}^{2}$, it follows that for any $t \in[0, T]$,

$$
\begin{aligned}
& e^{\eta t}\left|Y_{n}(t)\right|_{2}^{2}+\frac{1}{2} \sum_{k=1}^{N} \int_{t}^{T} e^{\eta s}\left|Z_{k, n}\right|_{2}^{2} d s \\
& \leq V_{T, n}-2 \sum_{k=1}^{N} \int_{t}^{T} \operatorname{Re} \int e^{\eta s} Y_{n} \overline{Z_{k, n}} d \xi d \beta_{k}(s),
\end{aligned}
$$


where

$$
\begin{aligned}
V_{T, n}:= & e^{\eta T}\left|X^{u}(T)-\mathbb{X}_{T}\right|_{2}^{2}+2 \gamma_{1} \int_{0}^{T} e^{\eta s}\left|X^{u}-\mathbb{X}_{1}\right|_{2}^{2} d s \\
& +\alpha|g|_{L^{\infty}} e^{\eta T} T^{\theta}\left\|X^{u}\right\|_{L^{q}\left(0, T ; L^{p}\right)}^{\alpha-1}\left\|Y_{n}\right\|_{L^{q}\left(0, T ; L^{p}\right)}^{2}
\end{aligned}
$$

This yields

$$
\begin{aligned}
\sum_{k=1}^{N} \mathbb{E}\left(\int_{0}^{T} e^{\eta s}\left|Z_{k, n}\right|_{2}^{2} d s\right)^{\frac{\rho_{\nu}}{2}} \\
\leq C\left(\rho_{\nu}\right)\left(\mathbb{E} V_{T, n}^{\frac{\rho_{\nu}}{2}}\right. \\
\left.\quad+\mathbb{E} \sup _{t \in[0, T]}\left|\sum_{k=1}^{N} \int_{0}^{t} \operatorname{Re} \int e^{\eta s} Y_{n} \overline{Z_{k, n}} d \xi d \beta_{k}(s)\right|^{\frac{\rho_{\nu}}{2}}\right) .
\end{aligned}
$$

Note that, $\mathbb{E} V_{T, n}^{\rho_{\nu} / 2}<\infty$, due to $(2.5)$, (4.31) and to the integrability conditions on $\mathbb{X}_{T}$ and $\mathbb{X}_{1}$. Moreover, by the Burkholder-Davis-Gundy inequality, the second term in the right-hand side of (4.43) is bounded by

$$
\begin{aligned}
C\left(\rho_{\nu}\right) & \mathbb{E}\left(\int_{0}^{T} \sum_{k=1}^{N}\left|\int e^{\eta s} Y_{n} \overline{Z_{k, n}} d \xi\right|^{2} d s\right)^{\frac{\rho_{\nu}}{4}} \\
\leq & C\left(\rho_{\nu}\right) \sum_{k=1}^{N} \mathbb{E} \sup _{t \in[0, T]} e^{\frac{1}{4} \rho_{\nu} \eta t}\left|Y_{n}(t)\right|_{2}^{\frac{\rho_{\nu}}{2}}\left(\int_{0}^{T} e^{\eta s}\left|Z_{k, n}\right|_{2}^{2} d s\right)^{\frac{\rho_{\nu}}{4}} \\
\leq & C\left(\rho_{\nu}, N\right) e^{\frac{1}{2} \rho_{\nu} \eta T} \mathbb{E}\left|Y_{n}\right|_{C\left([0, T] ; L^{2}\right)}^{\rho_{\nu}} \\
& +\frac{1}{2} \sum_{k=1}^{N} \mathbb{E}\left(\int_{0}^{T} e^{\eta s}\left|Z_{k, n}\right|_{2}^{2} d s\right)^{\frac{\rho_{\nu}}{2}} .
\end{aligned}
$$

Plugging this into (4.43), we get

$$
\begin{aligned}
\sup _{n \geq 1} \frac{1}{2} \sum_{k=1}^{N} \mathbb{E}\left(\int_{0}^{T} e^{\eta s}\left|Z_{k, n}\right|_{2}^{2} d s\right)^{\frac{\rho_{\nu}}{2}} \\
\leq C\left(\rho_{\nu}\right) \sup _{n \geq 1} \mathbb{E} V_{T, n}^{\frac{\rho_{\nu}}{2}} \\
\quad+C\left(\rho_{\nu}, N\right) e^{\frac{1}{2} \rho_{\nu} \eta T} \sup _{n \geq 1} \mathbb{E}\left|Y_{n}\right|_{C\left([0, T] ; L^{2}\right)}^{\rho_{\nu}}
\end{aligned}
$$

which by (4.31) implies (4.35), as claimed. 
Now, set

$$
\begin{aligned}
Y^{u}(\cdot):= & -\left(X^{u}(T)-\mathbb{X}_{T}\right)-\int_{.}^{T}\left(-i \Delta \tilde{Y}-\lambda i h_{1}\left(X^{u}\right) \tilde{Y}+\lambda i h_{2}\left(X^{u}\right) \overline{\widetilde{Y}}\right. \\
& \left.+\mu \widetilde{Y}-i V_{0} \tilde{Y}-i u \cdot V \tilde{Y}+\gamma_{1}\left(X^{u}-\mathbb{X}_{1}\right)-\sum_{k=1}^{N} \overline{\mu_{k}} e_{k} Z_{k}^{u}\right) d s \\
& -\sum_{k=1}^{N} \int_{.}^{T} Z_{k}^{u} d \beta_{k}(s) .
\end{aligned}
$$

By virtue of (4.32), (4.34), (4.36) and (4.37), we may pass to the limit in (4.24) and obtain that for any $v \in H^{2}, f \in L^{\infty}(\Omega \times(0, T))$,

$$
\mathbb{E} \int_{0}^{T} H^{-2}\langle\tilde{Y}(t), f(t) v\rangle_{H^{2}} d t=\mathbb{E} \int_{0}^{T} H^{-2}\left\langle Y^{u}(t), f(t) v\right\rangle_{H^{2}} d t,
$$

which implies that $Y^{u}=\tilde{Y}$, in $H^{-2}, d \mathbb{P} \otimes d t$-a.e. Since $Y^{u}$ is continuous in $H^{-2}$, $\mathbb{P}$-a.s., we can find a null set $N^{\prime}$, such that for any $\omega \notin N^{\prime},\left(Y^{u}(\omega), Z^{u}(\omega)\right)$ solves (2.9) in $H^{-2}$ for all $t \in[0, T]$, which proves the existence of solution to (2.9). Estimates (4.20) and (4.21) follow immediately by (4.31) and (4.35), respectively. Moreover, as in the proof of [1], Lemma 4.3, we have for $\left|Y^{u}(t)-Y^{u}(s)\right|_{2}^{2}$ an Itô formula similar to (4.38), which implies $t \rightarrow Y^{u}(t)$ is $L^{2}$ continuous.

The uniqueness can also be proved by duality arguments. Indeed, let $\left(Y_{j}^{u}, Z_{j}^{u}\right)$, $j=1,2$, be any two solutions to (2.9). For any $\Psi \in L^{\infty}\left(\Omega \times(0, T) \times \mathbb{R}^{d}\right)$, let $\psi$ be the unique solution to (4.26) but with $h_{j, n}\left(X^{u}\right)$ replaced by $h_{j}\left(X^{u}\right), j=1,2$. Define $\Lambda(\Psi)$ similarly as in (4.25) with $\psi_{n}$ replaced by $\psi$. Then, similar to (4.29), $\Lambda(\Psi)=\mathbb{E} \int_{0}^{T} \operatorname{Re}\left\langle\Psi, Y_{j}^{u}\right\rangle_{2} d t, j=1,2$. It follows that $Y_{1}^{u}=Y_{2}^{u}$ by the arbitrariness of $\Psi$, and so $Z_{1}^{u}=Z_{2}^{u}$ by an estimate similar to (4.44).

(ii). Fix $1 \leq j \leq N$. Consider the approximating equation of $\left(\partial_{j} Y^{u}, \partial_{j} Z^{u}\right)$ below $\left(\partial_{j}:=\frac{\partial}{\partial \xi_{j}}\right)$,

$$
\begin{aligned}
d Y_{n}^{\prime}= & -i \Delta Y_{n}^{\prime} d t+G_{n}\left(Y_{n}^{\prime}\right) d t \\
& -\sum_{k=1}^{N} \bar{\mu}_{k} e_{k} Z_{k, n}^{\prime} d t+\gamma_{1} \partial_{j}\left(X^{u}-\mathbb{X}_{1}\right) d t \\
& +F_{n}\left(X^{u}, Y^{u}, Z^{u}\right) d t+\sum_{k=1}^{N} Z_{k, n}^{\prime} d \beta_{k}(t), \\
Y_{n}^{\prime}= & -\left(\partial_{j} X^{u}(T)-\partial_{j} \mathbb{X}_{T}\right),
\end{aligned}
$$

where $X^{u}$ and $\left(Y^{u}, Z^{u}\right)$ are the solutions to (1.1) and (2.9), respectively,

$$
G_{n}\left(Y_{n}^{\prime}\right):=-\lambda i h_{1, n}\left(X^{u}\right) Y_{n}^{\prime}+\lambda i h_{2, n}\left(X^{u}\right) \overline{Y_{n}^{\prime}}+\left(\mu-i V_{0}-i u \cdot V\right) Y_{n}^{\prime},
$$




$$
\begin{aligned}
F_{n}\left(X^{u}, Y^{u}, Z^{u}\right):= & -\lambda i h_{1, n}^{\prime}\left(X^{u}\right) Y^{u}+\lambda i h_{2, n}^{\prime}\left(X^{u}\right) \overline{Y^{u}}+\partial_{j} \mu Y^{u}-i \partial_{j} V_{0} Y^{u} \\
& -i u \cdot \partial_{j} V Y^{u}-\sum_{k=1}^{N} \partial_{j}\left(\overline{\mu_{k}} e_{k}\right) Z_{k}^{u}
\end{aligned}
$$

$h_{k, n}\left(X^{u}\right)$ is as in (4.24) and $h_{k, n}^{\prime}\left(X^{u}\right)=g\left(\frac{\left|X^{u}\right|+\left|\nabla X^{u}\right|}{n}\right) \partial_{j}\left(h_{k}\left(X^{u}\right)\right), k=1,2$. By truncation, $\left|h_{k, n}\left(X^{u}\right)\right|+\left|h_{k, n}^{\prime}\left(X^{u}\right)\right| \leq C n^{\alpha-1}, k=1,2$, it follows that there exists a unique $\left(\mathcal{F}_{t}\right)$-adapted solution $\left(Y_{n}^{\prime}, Z_{n}^{\prime}\right) \in L^{2}\left(\Omega ; C\left([0, T] ; L^{2}\right)\right) \times\left(L_{\text {ad }}^{2}(0, T\right.$; $\left.\left.L^{2}\left(\Omega ; L^{2}\right)\right)\right)^{N}$ to $(4.46)$.

For each $\Psi \in L^{\infty}\left(\Omega \times(0, T) \times \mathbb{R}^{d}\right)$, let $\psi_{n}$ be the solution to (4.26). Similar to (4.25), set

$$
\begin{aligned}
\widetilde{\Lambda}_{j, n}(\Psi):= & \mathbb{E} \operatorname{Re}\left\langle\partial_{j} X^{u}(T)-\partial_{j} \mathbb{X}_{T}, \psi_{n}(T)\right\rangle_{2} \\
& \left.+\gamma_{1} \mathbb{E} \int_{0}^{T} \operatorname{Re}\left\langle\partial_{j} X^{u}(t)-\partial_{j} \mathbb{X}_{1}(t), \psi_{n}(t)\right)\right\rangle_{2} d t .
\end{aligned}
$$

By Itô's formula, we have

$$
\widetilde{\Lambda}_{j, n}(\Psi)=\mathbb{E} \int_{0}^{T} \operatorname{Re}\left(\Psi, Y_{n}^{\prime}\right\rangle_{2} d t-\mathbb{E} \int_{0}^{T} \operatorname{Re} \int F_{n}\left(X^{u}, Y^{u}, Z^{u}\right) \overline{\psi_{n}} d \xi d t
$$

Note that, since $\left|h_{1, n}^{\prime}\left(X^{u}\right)\right| \leq C\left|X^{u}\right|^{\alpha-2}\left|\partial_{j} X^{u}\right|$ and $2 \leq \alpha<1+4 / d$, by Hölder's inequality, we have for $(p, q)=\left(\alpha+1, \frac{4(\alpha+1)}{d(\alpha-1)}\right)$,

$$
\begin{aligned}
& \left|\int_{0}^{T} \operatorname{Re} \int(-\lambda i) h_{1, n}^{\prime}\left(X^{u}\right) Y^{u} \overline{\psi_{n}} d \xi d t\right| \\
& \quad \leq\left\|h_{1, n}^{\prime}\left(X^{u}\right) Y^{u}\right\|_{L^{q^{\prime}}\left(0, T ; L^{p^{\prime}}\right)}\left\|\psi_{n}\right\|_{L^{q}\left(0, T ; L^{p}\right)} \\
& \quad \leq C_{\alpha} T^{\theta}\left\|X^{u}\right\|_{L^{q}\left(0, T ; L^{p}\right)}^{\alpha-2}\left\|\partial_{j} X^{u}\right\|_{L^{q}\left(0, T ; L^{p}\right)}\left\|Y^{u}\right\|_{L^{q}\left(0, T ; L^{p}\right)}\left\|\psi_{n}\right\|_{L^{q}\left(0, T ; L^{p}\right)} \\
& \quad \leq C_{\alpha} T^{\theta}\left\|X^{u}\right\|_{L^{q}\left(0, T ; W^{1, p}\right)}^{\alpha-1}\left\|Y^{u}\right\|_{L^{q}\left(0, T ; L^{p}\right)}\left\|\psi_{n}\right\|_{L^{q}\left(0, T ; L^{p}\right)},
\end{aligned}
$$

where $\theta=1-d(\alpha-1) / 4 \in(0,1)$. Hence, for any $\rho \in\left[2, \rho_{v}\right)$,

$$
\begin{aligned}
\mid \mathbb{E} \int_{0}^{T} & \operatorname{Re} \int(-\lambda i) h_{1, n}^{\prime}\left(X^{u}\right) Y^{u} \overline{\psi_{n}} d \xi d t \mid \\
\leq & C(T) \mathbb{E}\left(\left\|X^{u}\right\|_{L^{q}\left(0, T ; W^{1, p}\right)}^{\alpha-1}\left\|Y^{u}\right\|_{L^{q}\left(0, T ; L^{p}\right)}\left\|\psi_{n}\right\|_{L^{q}\left(0, T ; L^{p}\right)}\right) \\
\leq & C(T)\left(\left\|X^{u}\right\|_{L^{\eta}\left(\Omega ; L^{q}\left(0, T ; W^{1, p}\right)\right)}^{\alpha-1}\right. \\
& \times\left\|Y^{u}\right\|_{L^{\rho_{\nu}\left(\Omega ; L^{q}\left(0, T ; L^{p}\right)\right)}}\left\|\psi_{n}\right\|_{\left.L^{\rho^{\prime}\left(\Omega ; L^{q}\left(0, T ; L^{p}\right)\right)}\right),}
\end{aligned}
$$

where $\eta$ satisfies $\frac{1}{(\alpha-1) \eta}=\frac{1}{\rho_{v}^{\prime}}-\frac{1}{\rho^{\prime}}>0$. Similar arguments apply to the term involving $\lambda i h_{2, n}^{\prime}\left(X^{u}\right) \overline{Y^{u}}$. Moreover, the other terms in the integration $\mathbb{E} \int_{0}^{T} \operatorname{Re} \int F_{n}\left(X^{u}\right.$, 
$\left.Y^{u}, Z^{u}\right) \overline{\psi_{n}} d \xi d t$ are bounded by

$$
C\left(\left\|Y^{u}\right\|_{L^{\rho}\left(\Omega ; L^{\infty}\left(0, T ; L^{2}\right)\right)}+\sum_{k=1}^{N}\left\|Z_{k}^{u}\right\|_{L^{\rho}\left(\Omega ; L^{2}\left(0, T ; L^{2}\right)\right)}\right)\left\|\psi_{n}\right\|_{L^{\rho^{\prime}\left(\Omega ; L^{\infty}\left(0, T ; L^{2}\right)\right)}} .
$$

Plugging the estimates above into (4.47) and using (4.28), (2.5), (4.20) and (4.21) we obtain for any $\rho \in\left[2, \rho_{\nu}\right)$,

$$
\begin{aligned}
\left|\mathbb{E} \int_{0}^{T} \operatorname{Re}\left\langle\Psi, Y_{n}^{\prime}\right\rangle_{2} d t\right| & \leq\left|\widetilde{\Lambda}_{j, n}(\Psi)\right|+\left|\mathbb{E} \int_{0}^{T} \operatorname{Re} \int F_{n}\left(X^{u}, Y^{u}, Z^{u}\right) \overline{\psi_{n}} d \xi d t\right| \\
& \leq C\|\Psi\|_{\mathcal{Y}_{\rho^{\prime}}^{\prime}}
\end{aligned}
$$

with $C$ independent of $n$ and $\Psi$, which implies that for any $\rho \in\left[2, \rho_{\nu}\right)$,

$$
\sup _{n \geq 1}\left\|Y_{n}^{\prime}\right\|_{\mathcal{Y}_{\rho}} \leq C
$$

Once we have obtained (4.49), using similar arguments as those below (4.31), we can prove the assertion (ii). The details are omitted.

Proof of Proposition 4.1. Using (4.5) in Lemma 4.2, we have

$$
\begin{aligned}
\lim _{\varepsilon \rightarrow 0} \frac{1}{\varepsilon}(\Phi(u+\varepsilon \tilde{u})-\Phi(u)) \\
=2 \mathbb{E}\left(\operatorname { R e } \left\langleX^{u}(T)-\mathbb{X}_{T},\left.\varphi^{u, \tilde{u}}(T)\right|_{2}\right.\right. \\
\left.\quad+\gamma_{1} \mathbb{E} \int_{0}^{T} \operatorname{Re}\left(X^{u}(t)-\mathbb{X}_{1}(t), \varphi^{u, \tilde{u}}(t)\right\rangle_{2} d t+\gamma_{2} \int_{0}^{T} u \cdot \tilde{u} d t\right) .
\end{aligned}
$$

Then, similar to (4.29), by (2.9) and (4.3), we obtain via Itô's formula,

$$
\begin{aligned}
& \mathbb{E} \operatorname{Re}\left\langle X^{u}(T)-\mathbb{X}_{T}, \varphi^{u, \tilde{u}}(T)\right\rangle_{2}+\gamma_{1} \mathbb{E} \int_{0}^{T} \operatorname{Re}\left\langle X^{u}(t)-\mathbb{X}_{1}(t), \varphi^{u, \tilde{u}}(t)\right\rangle_{2} d t \\
& =-\mathbb{E} \operatorname{Im} \int_{0}^{T} \int_{\mathbb{R}^{d}} \tilde{u} \cdot V X^{u} \overline{Y^{u}} d \xi d t
\end{aligned}
$$

Combining these formulas, we get (4.1) as claimed.

5. Proof of Theorem 2.6. As in the proof of Lemma 3.3, we note that $\Phi$ is continuous on the metric space $\mathcal{U}_{\mathrm{ad}}$ endowed with the distance $d(u, v)=\|u-v\|=$ $\left(\mathbb{E} \int_{0}^{T}|u(t)-v(t)|_{m}^{2} d t\right)^{1 / 2}$. Applying Ekeland's variational principle in $\mathcal{U}_{\text {ad }}$ (see [13], Theorem 1 , or [12]), for every $n \in \mathbb{N}$ we get $u_{n} \in \mathcal{U}_{\text {ad }}$ such that

$$
\Phi\left(u_{n}\right) \leq \Phi(u)+\frac{1}{n} d\left(u_{n}, u\right) \quad \forall u \in \mathcal{U}_{\mathrm{ad}} .
$$


In particular, it follows that

$$
u_{n}=\operatorname{argmin}\left\{\Phi(u)+\frac{1}{n}\left\|u_{n}-u\right\| ; u \in \mathcal{U}_{\mathrm{ad}}\right\} .
$$

We define the function $\widetilde{\Phi}: L_{\text {ad }}^{2}\left(0, T ; \mathbb{R}^{m}\right) \rightarrow \overline{\mathbb{R}}=(-\infty,+\infty]$ by

$$
\widetilde{\Phi}=\Phi(u)+I_{\mathcal{U}_{\mathrm{ad}}}(u) \quad \forall u \in L_{\mathrm{ad}}^{2}\left(0, T ; \mathbb{R}^{m}\right),
$$

where

$$
I_{\mathcal{U}_{\mathrm{ad}}}(u)= \begin{cases}0 & \text { if } u \in \mathcal{U}_{\mathrm{ad}} \\ +\infty & \text { otherwise }\end{cases}
$$

The subdifferential $\partial \widetilde{\Phi}(u) \subset L_{\text {ad }}^{2}\left(0, T ; \mathbb{R}^{m}\right)$ of $\widetilde{\Phi}$ at $u$ in the sense of R. T. Rockafellar [25] is defined as the set of all $z \in L_{\mathrm{ad}}^{2}\left(0, T ; \mathbb{R}^{m}\right)$ such that the function $v \rightarrow \widetilde{\Phi}(v)-\mathbb{E} \int_{0}^{T} v(t) z(t) d t$ has $u$ as a substationary point in the sense of [25].

We have

$$
\partial \widetilde{\Phi}(u) \subset \eta(u)+\mathcal{N}_{\mathcal{U}_{\mathrm{ad}}}(u) \quad \forall u \in \mathcal{U}_{\mathrm{ad}},
$$

where $\eta(u)$ is defined by (4.2), $\mathcal{N}_{\mathcal{U}_{\text {ad }}}(u)$ is the normal cone to $\mathcal{U}_{\text {ad }}$ at $u_{n}$, that is,

$$
\mathcal{N}_{\mathcal{U}_{\mathrm{ad}}}\left(u_{n}\right)=\left\{v \in L_{\mathrm{ad}}^{2}\left(0, T ; \mathbb{R}^{m}\right) ;\left\langle v, u_{n}-\widetilde{v}\right\rangle \geq 0, \forall \widetilde{v} \in \mathcal{U}_{\mathrm{ad}}\right\},
$$

and $\langle$,$\rangle denotes the inner product of L_{\mathrm{ad}}^{2}\left(0, T ; \mathbb{R}^{m}\right)$.

To prove (5.3), as mentioned in [25], (2.4), for each $u \in \mathcal{U}_{\mathrm{ad}}$, one has

$$
\partial \widetilde{\Phi}(u)=\left\{z \in L_{\mathrm{ad}}^{2}\left(0, T ; \mathbb{R}^{m}\right): \widetilde{\Phi}^{\uparrow}(u, y) \geq\langle y, z\rangle, \forall y \in L_{\mathrm{ad}}^{2}\left(0, T ; \mathbb{R}^{m}\right)\right\},
$$

where $\widetilde{\Phi}^{\uparrow}(u, y)$ is the subderivative at $u$ with respect to $y$

$$
\widetilde{\Phi}^{\uparrow}(u, y)=\sup _{V \subset \mathcal{N}(y)}\left[\limsup _{\substack{u^{\prime} \rightarrow u, \alpha^{\prime} \rightarrow \widetilde{\Phi}(u) \\ \alpha^{\prime} \geq \widetilde{\Phi}\left(u^{\prime}\right), t \rightarrow 0}} \inf _{y^{\prime} \in V}\left(\frac{\Phi\left(u^{\prime}+t y^{\prime}\right)-\alpha^{\prime}}{t}+\frac{I_{\mathcal{U}_{\mathrm{ad}}}\left(u^{\prime}+t y^{\prime}\right)}{t}\right)\right],
$$

and $\mathcal{N}(y)$ is the set of all neighborhoods of $y$. This yields

$$
\widetilde{\Phi}^{\uparrow}(u, y)=\lim _{t \rightarrow 0} \frac{\Phi(u+t y)-\Phi(u)}{t}+I_{\mathcal{U}_{\mathrm{ad}}}^{\prime}(u, y),
$$

where $I_{\mathcal{U}_{\mathrm{ad}}}^{\prime}(u, y)=0$ if $y \in T_{\mathcal{U}_{\mathrm{ad}}}(u)$, and $I_{\mathcal{U}_{\mathrm{ad}}}^{\prime}(u, y)=\infty$ if $y \notin T_{\mathcal{U}_{\mathrm{ad}}}(u), T_{\mathcal{U}_{\mathrm{ad}}}(u)$ is the (Clarke) tangent cone to $\mathcal{U}_{\text {ad }}$ at $u$ defined in [25]. Then, by Proposition 4.1, for any $z \in \partial \widetilde{\Phi}(u)$, we have that $\langle\eta(u), y\rangle \geq\langle y, z\rangle, \forall y=v-u, v \in \mathcal{U}_{\mathrm{ad}}$. Thus, $z \in \eta(u)+\mathcal{N}_{\mathcal{U}_{\mathrm{ad}}}(u)$. This implies (5.3).

On the other hand, by Theorem 2 in [25] we have

$$
\partial\left(\widetilde{\Phi}(u)+\frac{1}{n}\left\|u_{n}-u\right\|\right) \subset \partial \widetilde{\Phi}(u)+\frac{1}{n} \partial\left\|u_{n}-u\right\| .
$$


Thus, by (5.2)-(5.4) we get

$$
\begin{aligned}
0 & \in \partial\left(\widetilde{\Phi}(u)+\frac{1}{n}\left\|u_{n}-u\right\|\right)\left(u=u_{n}\right) \\
& \subset \eta\left(u_{n}\right)+\frac{1}{n}\left(\partial\left\|u_{n}-u\right\|\right)\left(u=u_{n}\right)+\mathcal{N}_{\mathcal{U}_{\mathrm{ad}}}\left(u_{n}\right),
\end{aligned}
$$

which implies that there exist $\zeta_{n} \in \mathcal{N}_{\mathcal{U}_{\mathrm{ad}}}\left(u_{n}\right)$ and $\eta_{n} \in\left(\partial\left\|u_{n}-u\right\|\right)\left(u=u_{n}\right)$, such that

$$
\eta\left(u_{n}\right)+\zeta_{n}+\frac{1}{n} \eta_{n}=0
$$

We claim that

$$
\mathcal{N}_{\mathcal{U}_{\mathrm{ad}}}\left(u_{n}\right)=\left\{v \in L_{\mathrm{ad}}^{2}\left(0, T ; \mathbb{R}^{m}\right): v \in N_{U}\left(u_{n}\right), \text { a.e. on }(0, T) \times \Omega\right\}
$$

where $N_{U}\left(u_{n}\right)$ is the normal cone to $U \subset \mathbb{R}^{m}$ at $u_{n} \in U$, that is, $N_{U}\left(u_{n}\right)=\{v \in$ $\left.\mathbb{R}^{m} ; v \cdot\left(u_{n}-\widetilde{v}\right) \geq 0, \forall \widetilde{v} \in U\right\}$.

Indeed, for any $\eta \in \mathcal{N}_{\mathcal{U}_{\mathrm{ad}}}\left(u_{n}\right)$, we have

$$
\mathbb{E} \int_{0}^{T} \eta \cdot\left(u_{n}-v\right) d t \geq 0 \quad \forall v \in \mathcal{U}_{\mathrm{ad}}
$$

Since for each closed convex set $U, \forall v>0,\left(I+v N_{U}\right)^{-1}=P_{U}$, where $P_{U}$ is the projection on $U$, there exists a unique $v \in \mathcal{U}_{\text {ad }}$, such that

$$
v+N_{U}(v) \ni u_{n}+\eta \quad \text { a.e. on }(0, T) \times \Omega,
$$

that is, $v=P_{U}\left(u_{n}+\eta\right)$, a.e. on $(0, T) \times \Omega$. Hence, there exists $\zeta_{v} \in N_{U}(v)$, such that $v+\zeta_{v}=u_{n}+\eta, d \mathbb{P} \otimes d t$-a.e. Then, by (5.7),

$$
0 \leq \mathbb{E} \int_{0}^{T}\left(v-u_{n}+\zeta_{v}\right) \cdot\left(u_{n}-v\right) d t=-\left\|u_{n}-v\right\|^{2}+\mathbb{E} \int_{0}^{T} \zeta_{v}\left(u_{n}-v\right) d t .
$$

Since $d \mathbb{P} \otimes d t$-a.e., $\zeta_{v} \in N_{U}(v), \zeta_{v} \cdot\left(v-u_{n}\right) \geq 0$, we get

$$
\left\|u_{n}-v\right\|^{2} \leq \mathbb{E} \int_{0}^{T} \zeta_{v} \cdot\left(u_{n}-v\right) d t \leq 0 .
$$

It follows that $u_{n}=v, d \mathbb{P} \otimes d t$-a.e., which yields by (5.8) that $\eta \in N_{U}\left(u_{n}\right), d \mathbb{P} \otimes$ $d t$-a.e., thereby implying

$$
\mathcal{N}_{\mathcal{U}_{\mathrm{ad}}}\left(u_{n}\right) \subset\left\{v \in L_{\mathrm{ad}}^{2}\left(0, T ; \mathbb{R}^{m}\right): v \in N_{U}\left(u_{n}\right) \text { a.e. on }(0, T) \times \Omega\right\} .
$$

The inverse inclusion is obvious. Thus we obtain (5.6), as claimed.

Now, by virtue of (4.2), we may rewrite (5.5) as

$$
\begin{aligned}
u_{n}(t)+\frac{1}{2 \gamma_{2}} \zeta_{n}^{0}(t)= & \frac{1}{\gamma_{2}} \operatorname{Im} \int_{\mathbb{R}^{d}} V(\xi) X_{n}(t, \xi) \overline{Y_{n}}(t, \xi) d \xi-\frac{1}{2 \gamma_{2} n} \eta_{n}(t) \\
& \text { a.e. on }(0, T) \times \Omega,
\end{aligned}
$$


where $\zeta_{n}^{0}(t) \in N_{U}\left(u_{n}(t)\right), X_{n}:=X^{u_{n}}$, and $\left(Y_{n}, Z_{n}\right)$ is the solution to (2.9) corresponding to $u_{n}$. We get by (5.9) that

$$
u_{n}(t)=P_{U}\left(\frac{1}{\gamma_{2}} \operatorname{Im} \int V(\xi) X_{n}(t, \xi) \overline{Y_{n}}(t, \xi) d \xi-\frac{1}{2 \gamma_{2} n} \eta_{n}(t)\right),
$$

with

$$
\mathbb{E} \int_{0}^{T}\left|\eta_{n}(t)\right|_{m}^{2} d t=1
$$

We claim that there exists a probability space $\left(\Omega^{*}, \mathcal{F}^{*}, \mathbb{P}^{*}\right), u_{n}^{*}, u^{*} \in \mathcal{U}_{\mathrm{ad}}, n \geq$ 1 , such that the distributions of $u_{n}^{*}$ and $u_{n}$ coincide on $L^{1}\left(0, T ; \mathbb{R}^{m}\right)$, and as $n \rightarrow$ $\infty$,

$$
u_{n}^{*} \rightarrow u^{*} \quad \text { in } L^{1}\left(0, T ; \mathbb{R}^{m}\right), \mathbb{P}^{*} \text {-a.s. }
$$

Then it follows from the boundedness of $\left\{u_{n}^{*}\right\}$ that

$$
u_{n}^{*} \rightarrow u^{*} \quad \text { in } L^{2}\left(0, T ; \mathbb{R}^{m}\right), \mathbb{P}^{*} \text {-a.s. }
$$

Hence, similar arguments as in the proof of Theorem 2.5 imply that

$$
\Phi^{*}\left(u^{*}\right)=\lim _{n \rightarrow \infty} \Phi^{*}\left(u_{n}^{*}\right)=\lim _{n \rightarrow \infty} \Phi\left(u_{n}\right)=I,
$$

thereby yielding the equality in (2.11).

It remains to prove (5.12). By virtue of Skorohod's representation theorem, we only need to show the tightness of the distributions of $u_{n}$ on $L^{1}\left(0, T ; \mathbb{R}^{m}\right), n \geq 1$. For this purpose, in view of Lemma A.2, it suffices to prove that $\mu_{n}:=\mathbb{P} \circ u_{n}^{-1}$, $n \geq 1$, satisfy (A.14) and (A.15).

Indeed, (A.14) follows immediately from the uniform boundedness of $\left\{u_{n}\right\}$. $\operatorname{Re}$ garding (A.15), by Markov's inequality, it suffices to show that there exists a positive exponent $b>0$ such that for any $\delta \in(0,1)$,

$$
\limsup _{n \rightarrow \infty} \mathbb{E} \sup _{0<h \leq \delta} \int_{0}^{T-h}\left|u_{n}(t+h)-u_{n}(t)\right|_{m} d t \leq C \delta^{b} .
$$

To this end, since $P_{U}$ is Lipschitz, using (5.10), the Cauchy inequality, (2.4) and (4.22) we get

$$
\begin{aligned}
\mathbb{E} \sup _{0<h \leq \delta} & \int_{0}^{T-h}\left|u_{n}(t+h)-u_{n}(t)\right|_{m} d t \\
\leq & \frac{1}{\gamma_{2} n} T^{\frac{1}{2}}+\frac{1}{\gamma_{2}}|V|_{L^{\infty}} \mathbb{E} \int_{0}^{T} \sup _{0<h \leq \delta}\left(\left|X_{n}(t+h)-X_{n}(t)\right|_{H^{-1}}\left|Y_{n}(t+h)\right|_{H^{1}}\right. \\
\quad & \left.+\left|Y_{n}(t+h)-Y_{n}(t)\right|_{H^{-1}}\left|X_{n}(t)\right|_{H^{1}}\right) d t \\
\leq & \frac{1}{\gamma_{2} n} T^{\frac{1}{2}}+C\left(\mathbb{E} \int_{0}^{T} \sup _{0<h \leq \delta}\left|X_{n}(t+h)-X_{n}(t)\right|_{H^{-1}}^{2} d t\right)^{\frac{1}{2}} \\
& +C\left(\mathbb{E} \int_{0}^{T} \sup _{0<h \leq \delta}\left|Y_{n}(t+h)-Y_{n}(t)\right|_{H^{-1}}^{2} d t\right)^{\frac{1}{2}} .
\end{aligned}
$$


Let us estimate $\mathbb{E} \int_{0}^{T} \sup _{0<h \leq \delta}\left|Y_{n}(t+h)-Y_{n}(t)\right|_{H^{-1}}^{2} d t$ on the right-hand side of (5.14). Similar arguments apply to $\mathbb{E} \int_{0}^{T} \sup _{0<h \leq \delta}\left|X_{n}(t+h)-X_{n}(t)\right|_{H^{-1}}^{2} d t$.

By the backward equation (2.9),

$$
\begin{aligned}
\mathbb{E} \int_{0}^{T} & \sup _{0<h \leq \delta}\left|Y_{n}(t+h)-Y_{n}(t)\right|_{H^{-1}}^{2} d t \\
\leq & \mathbb{E} \int_{0}^{T} \sup _{0<h \leq \delta}\left|\int_{t}^{t+h} i \Delta Y(s) d s\right|_{H^{-1}}^{2} d t \\
& +\mathbb{E} \int_{0}^{T} \sup _{0<h \leq \delta} \mid \int_{t}^{t+h}\left(-\lambda i h_{1}\left(X_{n}(s)\right) Y_{n}(s)\right.
\end{aligned}
$$

$$
\begin{aligned}
& \left.+\lambda i h_{2}\left(X_{n}(s)\right) \overline{Y_{n}}(s)\right)\left.d s\right|_{H^{-1}} ^{2} d t \\
& +\mathbb{E} \int_{0}^{T} \sup _{0<h \leq \delta}\left|\int_{t}^{t+h}\left(\mu-i V_{0}-i u_{n}(s) \cdot V\right) Y_{n}(s) d s\right|_{H^{-1}}^{2} d t \\
& +\mathbb{E} \int_{0}^{T} \sup _{0<h \leq \delta}\left|\int_{t}^{t+h}\left(\gamma_{1}\left(X_{n}(s)-\mathbb{X}_{1}(s)\right)-\sum_{k=1}^{N} \overline{\mu_{k}} e_{k} Z_{k, n}(s)\right) d s\right|_{H^{-1}}^{2} d t \\
& +\mathbb{E} \int_{0}^{T} \sup _{0<h \leq \delta}\left|\int_{t}^{t+h} \sum_{k=1}^{N} Z_{k, n}(s) d \beta_{k}(s)\right|_{H^{-1}}^{2} d t=: \sum_{j=1}^{5} K_{j} .
\end{aligned}
$$

For $K_{1}$, by (4.22),

$$
\begin{aligned}
K_{1} & \leq \mathbb{E} \int_{0}^{T} \sup _{0<h \leq \delta}\left(\int_{t}^{t+h}\left|Y_{n}(s)\right|_{H^{1}} d s\right)^{2} d t \\
& \leq \delta^{2} T \mathbb{E} \sup _{t \in[0, T+1]}\left|Y_{n}(t)\right|_{H^{1}}^{2} \leq C \delta^{2},
\end{aligned}
$$

where $C$ is independent of $n$.

Similarly, by Cauchy's inequality and by (4.20),

$$
\begin{aligned}
K_{3}+K_{4} \leq & \delta^{2} T\left(|\mu|_{\infty}+\left|V_{0}\right|_{\infty}+D_{U}\|V\|_{L^{\infty}\left(0, T+1 ; L^{\infty}\right)}\right) \mathbb{E} \sup _{t \in[0, T+1]}\left|Y_{n}(t)\right|_{2}^{2} \\
& +\delta \gamma_{1} \mathbb{E} \int_{0}^{T} \int_{t}^{t+\delta}\left|X_{n}(s)-\mathbb{X}_{1}(s)\right|_{2}^{2} d s d t \\
& +\delta \sum_{k=1}^{N}\left|\mu_{k}\right|\left|e_{k}\right|_{\infty} \mathbb{E} \int_{0}^{T} \int_{t}^{t+\delta}\left|Z_{k, n}(s)\right|_{2}^{2} d s d t \\
\leq & C\left(\delta+\delta^{2}\right),
\end{aligned}
$$

where $C$ is independent of $n$. 
Regarding $K_{2}$, choose the Strichartz pair $(p, q)=\left(\alpha+1, \frac{4(\alpha+1)}{d(\alpha-1)}\right)$. Since $p \in$ $\left(2, \frac{2 d}{d-2}\right), L^{p^{\prime}}\left(\mathbb{R}^{d}\right) \hookrightarrow H^{-1}\left(\mathbb{R}^{d}\right)$, we have

$$
\begin{aligned}
K_{2} & \leq \mathbb{E} \int_{0}^{T} \sup _{0<h \leq \delta}\left(\int_{t}^{t+h}\left|-\lambda i h_{1}\left(X_{n}(s)\right) Y_{n}(s)+\lambda i h_{2}\left(X_{n}(s)\right) \overline{Y_{n}}(s)\right|_{L^{p^{\prime}}} d s\right)^{2} d t \\
& \leq \alpha^{2} \mathbb{E} \int_{0}^{T} \sup _{0<h \leq \delta}\left(\int_{t}^{t+h}\left|X_{n}^{\alpha-1}(s) Y_{n}(s)\right|_{L^{p^{\prime}}} d s\right)^{2} d t \\
& \leq \delta^{2 / q} \alpha^{2} \mathbb{E} \int_{0}^{T}\left\|X_{n}^{\alpha-1} Y_{n}\right\|_{L^{q^{\prime}}\left(t, t+\delta ; L^{p^{\prime}}\right)}^{2} d t \\
& \leq \delta^{2 / q} \alpha^{2} T \mathbb{E}\left\|X_{n}^{\alpha-1} Y_{n}\right\|_{L^{q^{\prime}}\left(0, T+1 ; L^{p^{\prime}}\right)}^{2} .
\end{aligned}
$$

Note that, by Hölder's inequality,

$$
\left\|X_{n}^{\alpha-1} Y_{n}\right\|_{L^{q^{\prime}}\left(0, T ; L^{p^{\prime}}\right)} \leq T^{\theta}\left\|X_{n}\right\|_{L^{q}\left(0, T ; L^{p}\right)}^{\alpha-1}\left\|Y_{n}\right\|_{L^{q}\left(0, T ; L^{p}\right)},
$$

where $\theta=1-d(\alpha-1) / 4 \in(0,1)$. Hence,

$$
\begin{aligned}
K_{2} & \leq \delta^{2 / q} \alpha^{2} T^{2 \theta+1} \mathbb{E}\left\|X_{n}\right\|_{L^{q}\left(0, T ; L^{p}\right)}^{2(\alpha-1)}\left\|Y_{n}\right\|_{L^{q}\left(0, T+1 ; L^{p}\right)}^{2} \\
& \leq \delta^{2 / q} \alpha^{2} T^{2 \theta+1}\left\|X_{n}\right\|_{L^{\infty}\left(\Omega ; L^{q}\left(0, T ; L^{p}\right)\right)}^{2(\alpha-1)}\left\|Y_{n}\right\|_{L^{2}\left(\Omega ; L^{q}\left(0, T+1 ; L^{p}\right)\right)}^{2} .
\end{aligned}
$$

Then, by (2.5) and (4.20) we obtain

$$
K_{4} \leq C \delta^{2 / q}
$$

where $C$ is independent of $n$.

For $K_{5}$, using the Burkholder-Davis-Gundy inequality we get

$$
K_{5} \leq C \int_{0}^{T} \mathbb{E} \int_{t}^{t+\delta} \sum_{k=1}^{N}\left|Z_{k, n}(s)\right|_{2}^{2} d s d t .
$$

Then, using Fubini's theorem to interchange the sum and integrals, by (4.21) we have

$$
\begin{aligned}
K_{5} & \leq C \sum_{k=1}^{N} \mathbb{E}\left(\int_{0}^{\delta} \int_{0}^{s}+\int_{\delta}^{T} \int_{s-\delta}^{s}+\int_{T}^{T+\delta} \int_{s-\delta}^{T}\right)\left|Z_{k, n}(s)\right|_{2}^{2} d t d s \\
& \leq 3 \delta C \sum_{k=1}^{N} \mathbb{E} \int_{0}^{T+1}\left|Z_{k, n}(s)\right|_{2}^{2} d s \leq C \delta,
\end{aligned}
$$

where $C$ is independent of $n$.

Plugging (5.16)-(5.19) into (5.15), since $2 / q<1$ and $\delta<1$, we obtain

$$
\mathbb{E} \int_{0}^{T} \sup _{0<h \leq \delta}\left|Y_{n}(t+h)-Y_{n}(t)\right|_{H^{-1}}^{2} d t \leq C\left(\delta+\delta^{2}+\delta^{\frac{2}{q}}\right) \leq C \delta^{\frac{2}{q}},
$$

where $C$ is independent of $n$. 
The term $\mathbb{E} \int_{0}^{T} \sup _{0<h \leq \delta}\left|X_{n}(t+h)-X_{n}(t)\right|_{H^{-1}}^{2} d t$ in the right-hand side of (5.14) can be estimated similarly.

Therefore, in view of (5.14) we obtain (5.13) with $b=1 / q$, thereby proving the tightness of $\left\{\mu_{n}\right\}$ and yielding the equality in (2.11).

Finally, the stochastic maximum principle (2.12) follows from Proposition 4.1, taking into account that [see (5.5)] for the optimal $u^{*}, \eta\left(u^{*}\right)+\zeta^{*}=0$, where $\zeta^{*} \in \mathcal{N}_{\mathcal{U}_{\mathrm{ad}}}\left(u^{*}\right)$. Now, the proof is complete.

An example. We consider the case $m=1$ and $U=[0, \ell]$, where $\ell>0$. Then equation (2.12) reduces to

$$
u^{*}(t)= \begin{cases}0 & \text { if } \operatorname{Im} \int_{\mathbb{R}} V(\xi) X^{*}(t, \xi) \overline{Y^{*}}(t, \xi) d \xi \leq 0, \\ \frac{\ell}{\gamma_{2}} & \text { if } \operatorname{Im} \int_{\mathbb{R}} V(\xi) X^{*}(t, \xi) \overline{Y^{*}}(t, \xi) d \xi \geq \ell, \\ \frac{1}{\gamma_{2}} \operatorname{Im} \int_{\mathbb{R}} V(\xi) X^{*}(t, \xi) \overline{Y^{*}}(t, \xi) d \xi & \text { otherwise. }\end{cases}
$$

For the numerical computation of the optimal controller $u^{*}$, one can use the standard gradient descent algorithm suggested by (2.12). Namely,

$$
\begin{aligned}
u_{n+1}= & P_{U}\left(\frac{1}{1+2 \gamma_{2} \rho_{n}} u_{n}\right. \\
& \left.+\frac{2 \rho_{n}}{1+2 \gamma_{2} \rho_{n}} \operatorname{Im} \int_{\mathbb{R}^{d}} V(\xi) X_{n}(t, \xi) \bar{Y}_{n}(t, \xi) d \xi\right),
\end{aligned}
$$

where $\rho_{n}>0$ are suitably chosen and $X_{n}, Y_{n}$ are solutions to the forwardbackward system (1.1), (2.9) with $u=u_{n}$.

Proof of TheOREM 2.7. The proof is similar to that of Theorem 2.6. As a matter of fact, in the deterministic case, the analysis of the equation of variation and of the backward equation is much easier.

Similar to Lemma 4.2, we have

$$
\sup _{u, v \in \mathcal{U}_{\mathrm{ad}}}\left(\left\|\varphi^{u, \tilde{u}}\right\|_{C\left([0, T] ; H^{1}\right)}+\left\|\varphi^{u, \widetilde{u}}\right\|_{L^{q}\left(0, T ; W^{1, p}\right)}\right)<\infty,
$$

where $\tilde{u}=v-u, u, v \in \mathcal{U}_{\mathrm{ad}}$, and $\varphi^{u, \tilde{u}}$ is the solution to the deterministic equation of variation [i.e., (4.3) without $W]$. Moreover,

$$
\lim _{\varepsilon \rightarrow 0} \sup _{t \in[0, T]}\left|\varepsilon^{-1}\left(X^{u_{\varepsilon}}(t)-X^{u}(t)\right)-\varphi^{u, \tilde{u}}(t)\right|_{2}^{2} \rightarrow 0,
$$

where $X^{u_{\varepsilon}}$ and $X^{u}$ are the solutions to (1.1) corresponding to $u_{\varepsilon}:=u+\varepsilon \tilde{u}$ and $u$, respectively. 
Regarding the backward deterministic equation, we can now use time reversal arguments and the Strichartz estimates to obtain directly the estimate below:

$$
\sup _{u \in \mathcal{U}_{\mathrm{ad}}}\left(\left\|Y^{u}\right\|_{L^{\infty}\left(0, T ; H^{1}\right)}+\left\|Y^{u}\right\|_{L^{q}\left(0, T ; W^{1, p}\right)}\right)<\infty .
$$

Based on these, one also has the directional derivative of $\Phi$ as in Proposition 4.1, and similar to (5.13), the estimate below for the minimizing sequence of controls $\left\{u_{n}\right\}$ from Ekeland's principle,

$$
\limsup _{n \rightarrow \infty} \sup _{0<h \leq \delta} \int_{0}^{T-h}\left|u_{n}(t+h)-u_{n}(t)\right|_{m} d t \leq C \delta^{1 / q},
$$

which by the Riesz-Kolmogorov theorem implies that $\left\{u_{n}\right\}$ is relatively compact in $L^{1}\left(0, T ; \mathbb{R}^{m}\right)$, thereby yielding the result.

\section{APPENDIX}

Lemma A.1 ([26], Lemma 2.17). Let $T>0$ and $f \in C\left([0, T] ; \mathbb{R}_{+}\right)$, such that

$$
f \leq a+b f^{\alpha} \quad \text { on }[0, T]
$$

where $a, b>0, \alpha>1, a<\left(1-\frac{1}{\alpha}\right)(\alpha b)^{-\frac{1}{\alpha-1}}$, and $f(0) \leq(\alpha b)^{-\frac{1}{\alpha-1}}$. Then

$$
f \leq \frac{\alpha}{\alpha-1} a \quad \text { on }[0, T] \text {. }
$$

PROOF OF (2.4) AND (2.5). For simplicity, we omit the dependence of $u$ in $X^{u}$. We may assume $T \geq 1$ without loss of generality. Set

$$
H(X(t)):=\frac{1}{2}|\nabla X(t)|_{2}^{2}-\frac{\lambda}{\alpha+1}|X(t)|_{L^{\alpha+1}}^{\alpha+1} .
$$

As in the proof of [2], Theorem 3.1, we have for $t \in[0, T]$,

$$
\begin{aligned}
H(X(t)) & -H(x) \\
= & -\int_{0}^{t}\left(\operatorname{Im}\left\langle\left(\nabla V_{0}+u(s) \cdot \nabla V\right) X(s), \nabla X(s)\right\rangle\right) d s \\
& +\int_{0}^{t}\left(\operatorname{Re}|-\nabla(\mu X(s)), \nabla X(s)|_{2} d s+\frac{1}{2} \sum_{j=1}^{N}\left|\nabla\left(X(s) \phi_{j}\right)\right|_{2}^{2}\right) d s \\
& -\frac{1}{2} \lambda(\alpha-1) \sum_{j=1}^{N} \int_{0}^{t} \int\left(\operatorname{Re} \phi_{j}\right)^{2}|X(s)|^{\alpha+1} d \xi d s+M(t),
\end{aligned}
$$

where

$$
M(t):=\sum_{j=1}^{N} \int_{0}^{t}\left(\operatorname{Re}\left\langle\nabla\left(\phi_{j} X(s)\right),\left.\nabla X(s)\right|_{2}-\lambda \int \operatorname{Re} \phi_{j}|X(s)|^{\alpha+1} d \xi\right) d \beta_{j}(s) .\right.
$$


Below we shall treat the focusing and defocusing cases, respectively.

(i) (The focusing case $\lambda=1$.) Note that, by [2], Lemma 3.5,

$$
|X(t)|_{L^{\alpha+1}}^{\alpha+1} \leq C_{\varepsilon}|X(t)|_{2}^{p}+\varepsilon|\nabla X(t)|_{2}^{2},
$$

where $p=2 \frac{2(\alpha+1)-d(\alpha-1)}{4-d(\alpha-1)}>2$. As in the proof of [2], Theorem 3.7, the first three terms on the right-hand side of (A.2) are bounded by $C \int_{0}^{t}\left(|X(s)|_{2}^{p}+|X(s)|_{2}^{2}+\right.$ $\left.|\nabla X(s)|_{2}^{2}\right) d s$, where $C$ is independent of $u$. Thus, taking $\varepsilon<\frac{\alpha+1}{4}$ yields

$$
|\nabla X(t)|_{2}^{2} \leq 4 H(x)+C\left(|X(t)|_{2}^{p}+D(t)\right)+4 M(t),
$$

where $D(t):=\int_{0}^{t}\left(|X(s)|_{2}^{p}+|X(s)|_{2}^{2}+|\nabla X(s)|_{2}^{2}\right) d s$. It follows that for any $\rho \geq 4$,

$$
|\nabla X(t)|_{2}^{2 \rho} \leq C+C\left(|X(t)|_{2}^{\rho p}+D^{\rho}(t)+|M(t)|^{\rho}\right)
$$

with $C$ independent of $u$.

Note that, by Jensen's inequality and

$$
|X(t)|_{2}=|x|_{2}, \quad t \in[0, T],
$$

we have

$$
\begin{aligned}
\mathbb{E} \sup _{s \in[0, t]} D^{\rho}(s) & \leq \mathbb{E} \sup _{s \in[0, t]} s^{\rho-1} \int_{0}^{s}\left(|X(r)|_{2}^{p \rho}+|X(r)|_{2}^{2 \rho}+|\nabla X(r)|_{2}^{2 \rho}\right) d r \\
& \leq C(\rho, T)\left(1+\int_{0}^{t} \mathbb{E} \sup _{r \in[0, s]}|\nabla X(r)|_{2}^{2 \rho} d s\right) .
\end{aligned}
$$

Moreover, by the BDG inequality we get

$$
\begin{aligned}
\mathbb{E} \sup _{s \in[0, t]}|M(s)|^{\rho} \\
\leq C(\rho) \mathbb{E}\left(\int _ { 0 } ^ { t } \sum _ { j = 1 } ^ { N } \left(\left.\left|\operatorname{Re} / \nabla\left(\phi_{j} X(s)\right), \nabla X(s)\right|_{2}\right|^{2}\right.\right. \\
\left.\left.\quad+\left.\left.\left|\int \operatorname{Re} \phi_{j}\right| X(s)\right|^{\alpha+1} d \xi\right|^{2}\right) d s\right)^{\frac{\rho}{2}} \\
\leq C(\rho) \mathbb{E}\left(\int_{0}^{t}|\nabla X(s)|_{2}^{4}+|X(s)|_{2}^{4}+|X(s)|_{L^{\alpha+1}}^{2(\alpha+1)} d s\right)^{\frac{\rho}{2}} \\
\leq C(\rho, T) \mathbb{E} \int_{0}^{t}|\nabla X(s)|_{2}^{2 \rho}+|X(s)|_{2}^{2 \rho}+|X(s)|_{L^{\alpha+1}}^{(\alpha+1) \rho} d s .
\end{aligned}
$$

Then, using (A.6) and (A.3) one obtains the estimate

$$
\mathbb{E} \sup _{s \in[0, t]}|M(s)|^{\rho} \leq C(\rho, T)\left(1+\int_{0}^{t} \mathbb{E} \sup _{r \in[0, s]}|\nabla X(r)|_{2}^{2 \rho} d s\right) .
$$


Thus, plugging (A.7) and (A.9) into (A.5) and using (A.6) yield

$$
\mathbb{E} \sup _{s \in[0, t]}|\nabla X(s)|_{2}^{2 \rho} \leq C+C \int_{0}^{t} \sup _{r \in[0, s]}|\nabla X(r)|_{2}^{2 \rho} d s,
$$

which implies (2.4) by Gronwall's inequality.

(ii) (The defocusing case $\lambda=-1$.) Similar to (A.4), we have by (A.2),

$$
\begin{aligned}
& \frac{1}{2}|\nabla X(t)|_{2}^{2}+\frac{1}{\alpha+1}|X(t)|_{L^{\alpha+1}}^{\alpha+1} \\
& \quad \leq H(x)+C \int_{0}^{t}\left(|X(s)|_{2}^{2}+|\nabla X(s)|_{2}^{2}+|X(s)|_{L^{\alpha+1}}^{\alpha+1}\right) d s+M(t) .
\end{aligned}
$$

Using (A.6) and (A.8), we get for $\rho \geq 4$,

$$
\begin{aligned}
& \mathbb{E} \sup _{s \in[0, t]}\left(|\nabla X(t)|_{2}^{2 \rho}+|X(t)|_{L^{\alpha+1}}^{(\alpha+1) \rho}\right) \\
& \quad \leq C+C \int_{0}^{t} \mathbb{E} \sup _{r \in[0, s]}\left(|\nabla X(r)|_{2}^{2 \rho}+|X(r)|_{L^{\alpha+1}}^{(\alpha+1) \rho}\right) d s,
\end{aligned}
$$

and so (2.4) follows.

It remains to prove (2.5). Indeed, in the case where $e_{k}$ are constants, $1 \leq k \leq N$, by the rescaling transformation $y=e^{-W} X$, we have

$$
\partial_{t} y=-i \Delta y-\lambda i|y|^{\alpha-1} y+f(u) y,
$$

where $f(u):=-i\left(V_{0}+u \cdot V\right)$. Note that the Strichartz coefficient $C_{T}$ is now a deterministic constant. Then, arguing as in (3.19) we obtain that $\sup _{u \in \mathcal{U}_{\mathrm{ad}}}\left\|y^{u}\right\|_{L^{\infty}\left(\Omega ; L^{q}\left(0, T ; L^{p}\right)\right)}<\infty$ for any $\operatorname{Strichartz}$ pair $(p, q)$.

As regards the estimate for $\left\|X^{u}\right\|_{L^{\rho}\left(\Omega ; L^{q}\left(0, T ; W^{1, p}\right)\right)}$, it suffices to prove that for any $\rho \geq 1$,

$$
\sup _{u \in \mathcal{U}_{\mathrm{ad}}} \mathbb{E}\left\|y^{u}\right\|_{L^{q}\left(0, T ; W^{1, p}\right)}^{\rho}<\infty
$$

where $(p, q)=\left(\alpha+1, \frac{4(\alpha+1)}{d(\alpha-1)}\right)$.

Since $\left|\nabla\left(|y|^{\alpha-1} y\right)\right| \leq \alpha|y|^{\alpha-1}|\nabla y|,|y|_{L^{p}} \leq D|y|_{H^{1}}$, the Hölder inequality implies that (see, e.g., [2], (2.25))

(A.12) $\left\||y|^{\alpha-1} y\right\|_{L^{q^{\prime}}\left(0, t ; W^{1, p^{\prime}}\right)} \leq 2 \alpha D^{\alpha-1} t^{\theta}\|y\|_{C\left([0, T] ; H^{1}\right)}^{\alpha-1}\|y\|_{L^{q}\left(0, t ; W^{1, p}\right)}$,

where $\theta=1-2 / q>0$. Moreover,

$$
\|f(u) y\|_{L^{1}\left(0, t ; H^{1}\right)} \leq T\|f(u)\|_{L^{\infty}\left(0, T ; W^{1, \infty}\right)}\|y\|_{C\left([0, t] ; H^{1}\right)} .
$$


Thus, applying Strichartz estimates to (A.10) and using the estimates above, we get

$\|y\|_{L^{q}\left(0, t ; W^{1, p}\right)}$

(A.13)

$$
\begin{aligned}
\leq & C\left(|x|_{H^{1}}+2 \alpha D^{\alpha-1} t^{\theta}\|y\|_{C\left([0, T] ; H^{1}\right)}^{\alpha-1}\|y\|_{L^{q}\left(0, t ; W^{1, p}\right)}\right. \\
& \left.+T\|f(u)\|_{L^{\infty}\left(0, T ; W^{1, \infty}\right)}\|y\|_{C\left([0, t] ; H^{1}\right)}\right) \\
\leq & D(T)\left(\|y\|_{C\left([0, T] ; H^{1}\right)}+t^{\theta}\|y\|_{L^{q}\left(0, t ; W^{1, p}\right)}\right)
\end{aligned}
$$

where

$$
D(T)=1+2 \alpha D^{\alpha-1}\|y\|_{C\left([0, T] ; H^{1}\right)}^{\alpha-1}+T \sup _{u \in \mathcal{U}_{\mathrm{ad}}}\|f(u)\|_{L^{\infty}\left(\Omega ; L^{\infty}\left(0, T ; W^{1, \infty}\right)\right)} .
$$

Then, similar to (3.19) we have

$$
\left\|y^{u}\right\|_{L^{q}\left(0, T ; W^{1, p}\right)} \leq 2\left(\left[\frac{T}{t}\right]+1\right)^{\frac{1}{q}} D(T)\left\|y^{u}\right\|_{C\left([0, T] ; H^{1}\right)},
$$

where $t=2^{-1 / \theta} D(T)^{-1 / \theta} \wedge T$.

Therefore, taking into account (2.4) we obtain (A.11), thereby completing the proof.

LEMMA A.2. Let $\mu_{n}, n \geq 1$, be a family of probability measures on $L^{1}\left(0, T ; \mathbb{R}^{m}\right)$. Assume that

(A.14) $\lim _{R \rightarrow \infty} \limsup _{n \rightarrow \infty} \mu_{n}\left\{v \in L^{1}\left(0, T ; \mathbb{R}^{m}\right): \int_{0}^{T}|v(t)|_{m} d t>R\right\}=0$

and for any $\varepsilon>0$,

$$
\begin{aligned}
\lim _{\delta \rightarrow 0} & \limsup _{n \rightarrow \infty} \mu_{n}\left\{v \in L^{1}\left(0, T ; \mathbb{R}^{m}\right):\right. \\
& \left.\sup _{0<h \leq \delta} \int_{0}^{T-h}|v(t+h)-v(t)|_{m} d t>\varepsilon\right\}=0 .
\end{aligned}
$$

Then $\left\{\mu_{n}\right\}_{n \geq 1}$ is tight on $L^{1}\left(0, T ; \mathbb{R}^{m}\right)$.

Proof. Set $K_{1}(R)=\left\{v \in L^{1}\left(0, T ; \mathbb{R}^{m}\right): \int_{0}^{T}|v(t)|_{m} d t \leq R\right\}$ and $K_{2}(\delta, \varepsilon)=$ $\left\{v \in L^{1}\left(0, T ; \mathbb{R}^{m}\right): \sup _{0<h \leq \delta} \int_{0}^{T-h}|v(t+h)-v(t)|_{m} d t \leq \varepsilon\right\}$, where $R, \delta, \varepsilon>0$.

Fix $\varepsilon>0$. By (A.14), there exists $N(=N(\varepsilon)), R_{1}\left(=R_{1}(\varepsilon)\right) \geq 1$, such that $\sup _{n \geq N} \mu_{n}\left(K_{1}^{c}\left(R_{1}\right)\right) \leq \frac{\varepsilon}{2}$. Since for each $n \geq 1, \lim _{R \rightarrow \infty} \mu_{n}\left(K_{1}^{c}(R)\right)=0$, we can choose $R_{2}\left(=R_{2}(\varepsilon)\right)$ sufficiently large, such that $\sup _{1 \leq n \leq N} \mu_{n}\left(K_{1}^{c}\left(R_{2}\right)\right) \leq \frac{\varepsilon}{2 N}$. Thus, letting $R=R_{1} \vee R_{2}$ we get $\sup _{n \geq 1} \mu_{n}\left(K_{1}^{c}(R)\right) \leq \varepsilon$.

Similarly, since for each $k, n \geq 1, \lim _{\delta \rightarrow 0} \mu_{n}\left(K_{2}^{c}\left(\delta, \frac{1}{k}\right)\right)=0$, by (A.15) and similar arguments as above, we can choose $\delta_{k}>0$ sufficiently small such that $\sup _{n \geq 1} \mu_{n}\left(K_{2}^{c}\left(\delta_{k}, \frac{1}{k}\right)\right) \leq \frac{\varepsilon}{2^{k}}$. 
Then set $K:=K_{1}(R) \cap\left(\bigcap_{k \geq 1} K_{2}\left(\delta_{k}, \frac{1}{k}\right)\right)$. It follows from [27], Theorem 1, that $K$ is relatively compact in $L^{1}\left(0, T ; \mathbb{R}^{m}\right)$, and by the estimates above we have $\sup _{n \geq 1} \mu_{n}\left(K^{c}\right) \leq 2 \varepsilon$, which implies the tightness of $\left\{\mu_{n}\right\}_{n \geq 1}$ on $L^{1}\left(0, T ; \mathbb{R}^{m}\right)$.

Acknowledgment. We thank the referee for helpful comments.

\section{REFERENCES}

[1] BARbu, V., Röckner, M. and Zhang, D. (2014). Stochastic nonlinear Schrödinger equations with linear multiplicative noise: Rescaling approach. J. Nonlinear Sci. 24 383-409. MR3215081

[2] Barbu, V., Röckner, M. and Zhang, D. (2016). Stochastic nonlinear Schrödinger equations. Nonlinear Anal. 136 168-194. MR3474409

[3] Barbu, V., Röckner, M. and Zhang, D. (2017). Stochastic nonlinear Schrödinger equations: No blow-up in the non-conservative case. J. Differential Equations 263 7919-7940. MR3705702

[4] Barchielli, A. and Gregoratti, M. (2009). Quantum Trajectories and Measurements in Continuous Time. The Diffusive Case. Lecture Notes in Physics 782. Springer, Heidelberg. MR2841028

[5] Beauchard, K., Coron, J. M., Mirrahimi, M. and Rouchon, P. (2007). Implicit Lyapunov control of finite dimensional Schrödinger equations. Systems Control Lett. 56388 395. MR2311201

[6] Borzì, A. and Hohenester, U. (2007/08). Multigrid optimization schemes for solving Bose-Einstein condensate control problems. SIAM J. Sci. Comput. 30 441-462. MR2377449

[7] Butkovskiy, A. G. and Samoylenko, Yu. I. (1990). Control of Quantum-Mechanical Processes and Systems. Mathematics and Its Applications (Soviet Series) 56. Kluwer Academic, Dordrecht. MR1070712

[8] Da Prato, G. and ZabczyK, J. (2014). Stochastic Equations in Infinite Dimensions, 2nd ed. Encyclopedia of Mathematics and Its Applications 152. Cambridge Univ. Press, Cambridge. MR3236753

[9] De Bouard, A. and Debussche, A. (1999). A stochastic nonlinear Schrödinger equation with multiplicative noise. Comm. Math. Phys. 205 161-181. MR1706888

[10] DE Bouard, A. and Debussche, A. (2003). The stochastic nonlinear Schrödinger equation in $H^{1}$. Stoch. Anal. Appl. 21 97-126. MR1954077

[11] Edelstein, M. (1968). On nearest points of sets in uniformly convex Banach spaces. J. Lond. Math. Soc. (2) 43 375-377. MR0226364

[12] Ekeland, I. (1974). On the variational principle. J. Math. Anal. Appl. 47 324-353. MR0346619

[13] Ekeland, I. (1979). Nonconvex minimization problems. Bull. Amer. Math. Soc. (N.S.) $1443-$ 474. MR0526967

[14] Fuhrman, M. and Tessitore, G. (2002). Nonlinear Kolmogorov equations in infinite dimensional spaces: The backward stochastic differential equations approach and applications to optimal control. Ann. Probab. 30 1397-1465. MR1920272

[15] GYÖNGY, I. and KRYLOV, N. (1996). Existence of strong solutions for Itô's stochastic equations via approximations. Probab. Theory Related Fields 105 143-158. MR1392450

[16] Hintermüller, M., Marahrens, D., Markowich, P. A. and Sparber, C. (2013). Optimal bilinear control of Gross-Pitaevskii equations. SIAM J. Control Optim. 51 25092543. MR3069086 
[17] HU, Y. and Peng, S. G. (1991). Adapted solution of a backward semilinear stochastic evolution equation. Stoch. Anal. Appl. 9 445-459. MR1133858

[18] ITO, K. and KUNISCH, K. (2007). Optimal bilinear control of an abstract Schrödinger equation. SIAM J. Control Optim. 46 274-287. MR2299629

[19] JäGer, G., Reich, D. M., Goerz, M. H., Koch, C. P. and Hohenester, U. (2014). Optimal quantum control of Bose-Einstein condensates in magnetic microtrps: Comparison of gradient-ascent-pulse-engineering and Krotov optimization schemes. Phys. Rev. A 90 033628 .

[20] Linares, F. and Ponce, G. (2009). Introduction to Nonlinear Dispersive Equations. Springer, New York. MR2492151

[21] LiU, W. and Röckner, M. (2015). Stochastic Partial Differential Equations: An Introduction. Springer, Cham. MR3410409

[22] Marzuola, J., Metcalfe, J. and Tataru, D. (2008). Strichartz estimates and local smoothing estimates for asymptotically flat Schrödinger equations. J. Funct. Anal. 255 1497-1553. MR2565717

[23] Mirrahimi, M., Rouchon, P. and Turinici, G. (2005). Lyapunov control of bilinear Schrödinger equations. Automatica J. IFAC 41 1987-1994. MR2168664

[24] Peirce, A. P., Dahleh, M. A. and Rabitz, H. (1988). Optimal control of quantummechanical systems: Existence, numerical approximation, and applications. Phys. Rev. A (3) 37 4950-4964. MR0949169

[25] Rockafellar, R. T. (1979). Directionally Lipschitzian functions and subdifferential calculus. Proc. Lond. Math. Soc. (3) 39 331-355. MR0548983

[26] SaAnouni, T. (2015). Remarks on damped fractional Schrödinger equation with pure power nonlinearity. J. Math. Phys. 56 061502, 14. MR3369869

[27] Simon, J. (1987). Compact sets in the space $L^{p}(0, T ; B)$. Ann. Mat. Pura Appl. (4) 146 65-96. MR0916688

[28] Tessitore, G. (1996). Existence, uniqueness and space regularity of the adapted solutions of a backward SPDE. Stoch. Anal. Appl. 14 461-486. MR1402690

[29] Werschnik, J. and Gross, E. K. U. (2007). Quantum optimal control theory. J. Phys. B 40 R175-R211. MR2355901

[30] Yong, J. and ZHou, X. Y. (1999). Stochastic Controls. Hamiltonian Systems and HJB Equations. Applications of Mathematics (New York) 43. Springer, New York. MR1696772

\section{BARBU}

OCTAV MAYER INSTITUTE OF MATHEMATICS

(ROMANIAN ACADEMY)

AND

AL.I. CUZA UNIVERSITY

700506, IAŞI

ROMANIA

E-MAIL: vb41@uaic.ro

\author{
M. RÖCKNER \\ FAKULTÄT FÜR MATHEMATIK \\ UNIVERSITÄT BIELEFELD \\ D-33501 BIELEFELD \\ GERMANY \\ E-MAIL: roeckner@math.uni-bielefeld.de
}

\author{
D. ZHANG \\ SCHOOL OF Mathematical SCIENCES \\ Shanghai JiaO TONG UNIVERSiTY \\ 200240 SHANGHAI \\ CHINA \\ AND \\ FAKULTÄT FÜR MATHEMATIK \\ UNIVERSITÄT BIELEFELD \\ D-33501 BIELEFELD \\ GERMANY \\ E-MAIL: dzhang@sjtu.edu.cn
}

